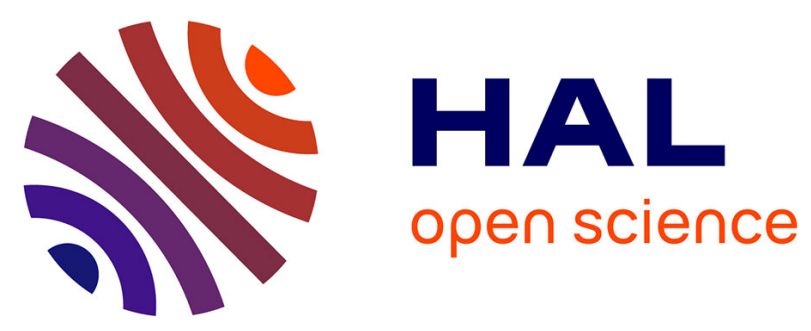

\title{
Scalable Disordered Hyperuniform Architectures via Nanoimprint Lithography of Metal Oxides
}

Zeinab Chehadi, Mohammed Bouabdellaoui, Mehrnaz Modaresialam, Thomas Bottein, Marco Salvalaglio, Monica Bollani, David Grosso, Marco Abbarchi

\section{- To cite this version:}

Zeinab Chehadi, Mohammed Bouabdellaoui, Mehrnaz Modaresialam, Thomas Bottein, Marco Salvalaglio, et al.. Scalable Disordered Hyperuniform Architectures via Nanoimprint Lithography of Metal Oxides. ACS Applied Materials \& Interfaces, 2021, 13 (31), pp.37761-37774. 10.1021/acsami.1c05779 . hal-03328593

\section{HAL Id: hal-03328593 \\ https://hal.science/hal-03328593}

Submitted on 31 Aug 2021

HAL is a multi-disciplinary open access archive for the deposit and dissemination of scientific research documents, whether they are published or not. The documents may come from teaching and research institutions in France or abroad, or from public or private research centers.
L'archive ouverte pluridisciplinaire HAL, est destinée au dépôt et à la diffusion de documents scientifiques de niveau recherche, publiés ou non, émanant des établissements d'enseignement et de recherche français ou étrangers, des laboratoires publics ou privés. 


\section{Scalable Disordered Hyperuniform Architectures}

\section{Via Nano-Imprint Lithography Of Metal Oxides}

Zeinab Chehadi ${ }^{l}$, Mohammed Bouabdellaoui ${ }^{1}$, Mehrnaz Modaresialam ${ }^{l}$, Thomas Bottein ${ }^{1, \hat{*}}$,

Marco Salvalaglio ${ }^{2,3}$, Monica Bollani ${ }^{4}$, David Grosso $^{1}$, and Marco Abbarchi ${ }^{1, *}$

${ }^{1}$ Aix Marseille Univ, Université de Toulon, CNRS, IM2NP Marseille, France

${ }^{2}$ Institute of Scientific Computing, TU Dresden, 01062 Dresden, Germany

${ }^{3}$ Dresden Center for Computational Materials Science (DCMS), TU Dresden, 01062 Dresden, Germany

${ }^{4}$ Istituto di Fotonica e Nanotecnologie-Consiglio Nazionale delle Ricerche, Laboratory for Nanostructure Epitaxy and Spintronics on Silicon, Via Anzani 42, 22100 Como, Italy

KEYWORDS. Disordered hyperuniform materials, Sol-gel dip coating, Nano-imprint lithography, titania, silica. 


\section{ABSTRACT}

Fabrication and scaling of disordered hyperuniform materials remain hampered by the difficulties in controlling the spontaneous phenomena leading to this novel kind of exotic arrangement of objects. Here we demonstrate a hybrid top-down/bottom-up approach based on sol-gel dip-coating and nano-imprint lithography for the faithful reproduction of disordered hyperuniform metasurfaces in metal oxides. Nano- to micro-structures made of silica and titania can be directly printed over several $\mathrm{cm}^{2}$ on glass and on silicon substrates. First, we describe the polymer mold fabrication starting from a hard master obtained via spontaneous solid-state dewetting of SiGe and Ge thin layers on $\mathrm{SiO}_{2}$. Then we assess the effective disordered hyperuniform character of master and replica and the role of the thickness of the sol-gel layer on the metal oxide replicas and on the presence of a residual layer underneath. Finally, as a potential application, we show the antireflective character of titania structures on silicon. Our results are relevant for the realistic implementation over large scales of disordered hyperuniform nano- and micro-architectures made of metal oxides thus opening their exploitation in the framework of wet chemical assembly.

\section{INTRODUCTION}

Disordered hyperuniform ( $\mathrm{dHU}$ ) materials are an emerging class of amorphous systems at the edge between ordered crystals (as they do not display long-range density fluctuations) and liquids (as they do not have Bragg peaks in diffraction). This idea was theoretically introduced about

twenty years ago by S. Torquato and F. H Stillinger ${ }^{1}$ and its comprehension and exploitation in practical applications are still at their beginning. In the last years, dHU features have been 
highlighted in a variety of natural phenomena spanning from classical ${ }^{2,3}$ to quantum systems ${ }^{4-6}$, from out-of-equilibrium at room temperature ${ }^{7}$ to in equilibrium ${ }^{8,9}$ including biological frameworks ${ }^{10}$. Beyond fundamental understanding of natural phenomena, the interest in this peculiar class of correlated disorder springs from the potential applications it may have in anomalous light and matter transport. Generally speaking, disordered materials are less prone to fabrication imperfections with respect to the ordered counterparts while displaying spatial steadiness of the photonic modes ${ }^{11}$. As such, if obtained via self-assembly over large scales, they may be much less expensive. In optics (e.g. for anti-reflection coatings) another advantage of disordered structures with respect to ordered arrays of objects (e.g. such as moth-eye structures for anti-reflection coatings), is the lack of diffraction from which neat colorization and angular dependencies can spring ${ }^{12}$.

Artificial structures, such as waveguide polarizers ${ }^{13}$, lasers ${ }^{14}$, metalenses ${ }^{15}$, photonic band gaps for light propagation ${ }^{16-18}$ plasmonic metasurfaces ${ }^{19}$, and efficient light trapping in thin Si films ${ }^{20}$ are recent examples of dHU systems, typically obtained via top-down fabrication methods or hybrid self-assembly and top-down ${ }^{21}$. Nevertheless, dHU systems can be, in principle, obtained by self-assembly, exploiting advection ${ }^{22}$, emulsions ${ }^{23}$, block copolymers assembly ${ }^{23}$, and sedimentation ${ }^{24,25}$. Other remarkable examples of $\mathrm{dHU}$ materials are those obtained via spontaneous pattern formation in systems following Swift-Hohenberg ${ }^{26,27}$ and Cahn-Hilliard ${ }^{28}$ dynamics (respectively describing wrinkling and phase separation via spinodal decomposition) as recently pointed out by $\mathrm{Ma}$ and coworkers ${ }^{29,30}$. These spontaneous phenomena are mainly observed in liquid and soft materials.

Very recently, spinodal-like solid-state dewetting of $\mathrm{SiGe}$ alloys on $\mathrm{SiO}_{2}$ was demonstrated as an alternative, self-assembly method for the fabrication of high-dielectric constant, effectively 
dHU metasurfaces ${ }^{31}$. The advantage of this approach is the lack of constraints in the extension of the dewetted wafers ${ }^{32}$ that, in principle, can be extended up to 8 inches using state-of-the-art molecular beam epitaxy reactors. Nevertheless, the implementation of dHU architectures made of such hard materials and having a typical size that can be tuned at will from tens of nm up to tens of $\mu \mathrm{m}$ can be conveniently exploited as masters for nano-imprint lithography ${ }^{33}$.

Here we show that SiGe-based micro- and nano-architectures can be faithfully reproduced in high- and low-dielectric constant metal oxides such as titania and silica, on silicon and glass wafers combining sol-gel dip-coating and nano-imprint lithography (soft-NIL). First, we describe shortly the SiGe master preparation via spinodal-like solid-state dewetting. Then, we discuss the differences in the polymeric mold preparation starting from a SiGe master with respect to conventional Si-based ones. We provide a detailed description of the sol-gel formulations and of the nano-imprint lithography process. We address the effective dHU character of the masters and of the metal oxide replicas by measuring their bidirectional reflectance distribution function under laser and white light illumination thus assessing the properties of the corresponding structure factor $\mathrm{S}(\mathrm{k})$. Note that, differently with previous investigations on these systems, here we directly measure the dHU character through light scattering rather than inferring it from the analysis of the morphology emerging patterns, thus addressing distinctive features of this class of disordered materials. Finally, study the effect of the initial sol-gel layer thickness on the quality of the metal oxide replicas and show its influence on the properties of our dHU metasurfaces as anti-reflection coatings on silicon.

\section{EXPERIMENTAL SECTION}

\section{MASTER PREPARATION}



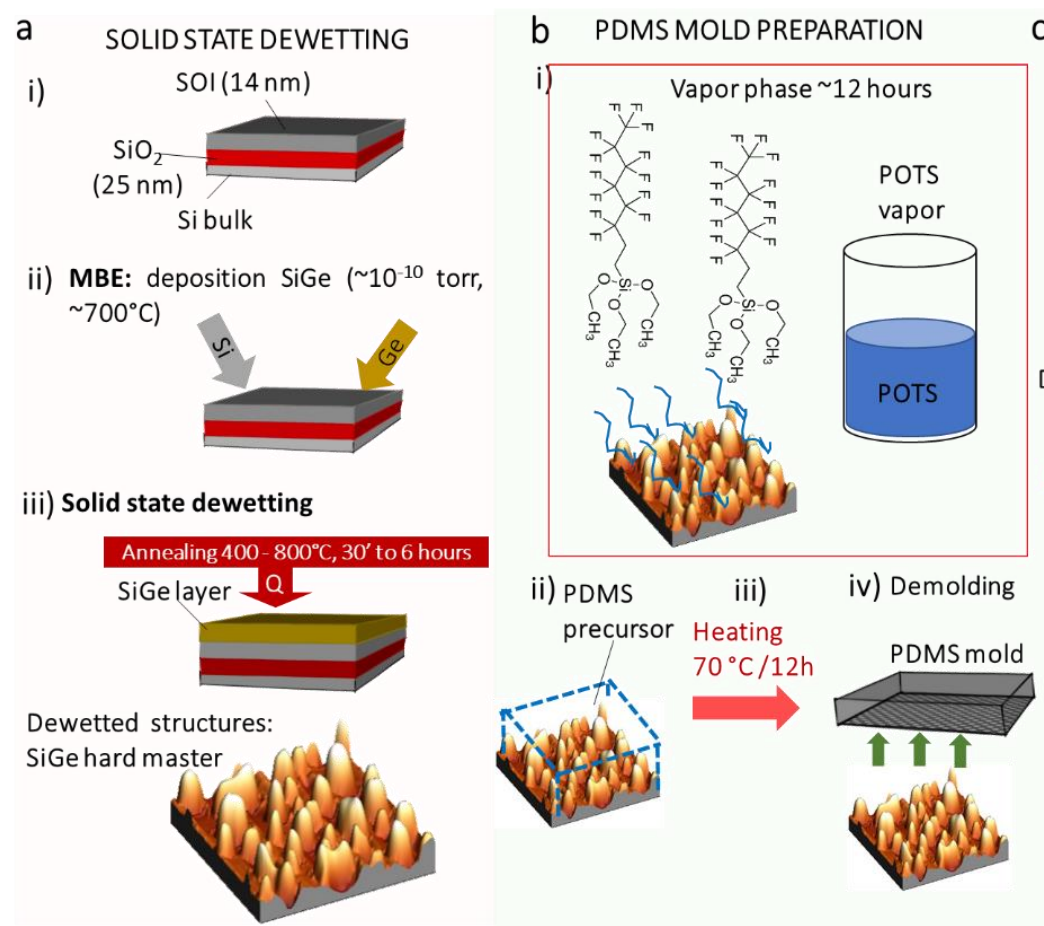
C SOL-GELDIP COATING AND NANO-IMPRINT
LITHOGRAPHY
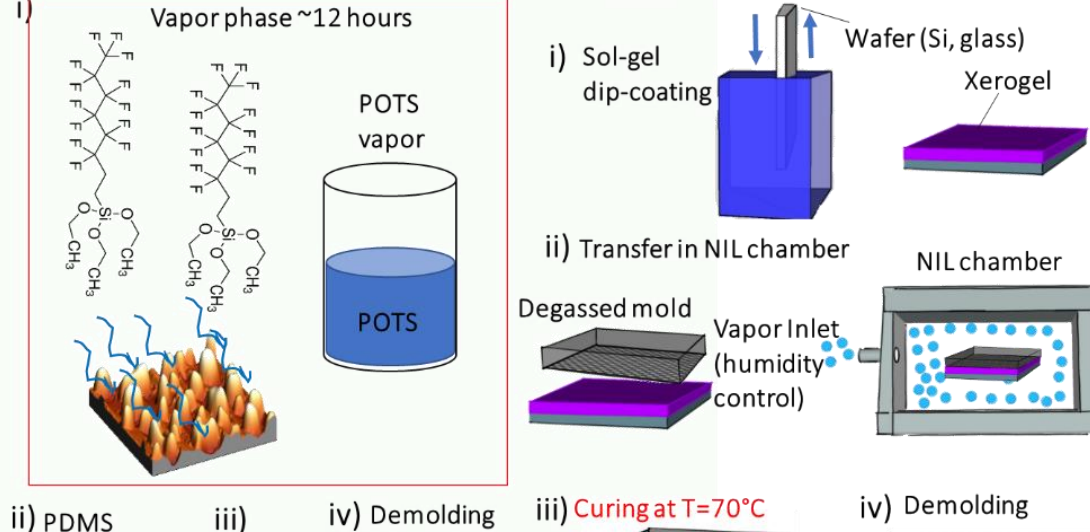

ii) Transfer in NIL chamber

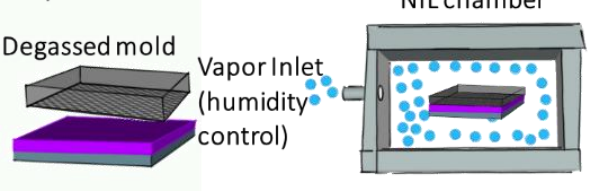

ii) PDMS

iii)

iv) Demolding

iii) Curing at $\mathrm{T}=70^{\circ} \mathrm{C}$

iv) Demolding
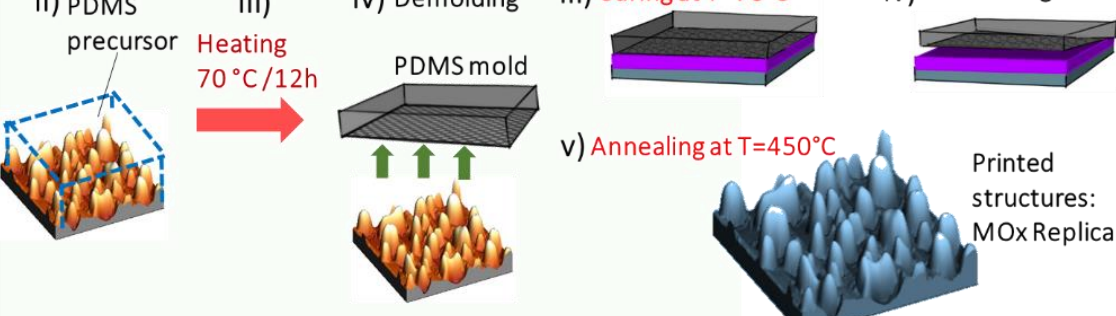

v) Annealing at $T=450^{\circ} \mathrm{C}$

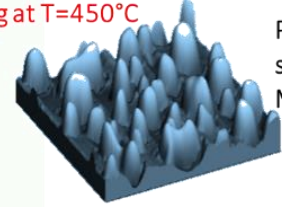

Printed structures: MOx Replica

Figure 1. (a) Elaboration of hard masters by solid state dewetting. (b) Preparation of PDMS mold. (c) Dip coating of metal oxide sol gel film and transfer of dewetted structures by nanoimprint lithography on sol-gel film.

The hard masters used in this work were elaborated by a self-assembly method based on solid state dewetting of ultra-thin silicon films on insulator (UT-SOI) and SiGe (or Ge) deposition via molecular beam epitaxy (Figure 1a) ${ }^{9,34}$. The UT-SOI substrates used here were commercial monocrystalline Si films, $14 \mathrm{~nm}$ thick, (001)-oriented on $25 \mathrm{~nm}$ thick buried oxide on a Si (001) substrate. All the samples in use were diced in squares of $2 \mathrm{~cm} \times 2 \mathrm{~cm}$ in size. The surface preparation for epitaxy consists of ex-situ and in-situ cleaning. Ex-situ chemical cleaning consisted of: 1) 10' in acetone in ultrasonic bath; 2) 10' Ethanol in ultrasonic bath; 3) 10' in $\mathrm{NO}_{2}$ plasma; 4) 30 ' in a solution of $10 \%$ HF-deionized $\mathrm{H}_{2} \mathrm{O}$ in $\mathrm{N}_{2}$ atmosphere in order to leave the surface passivated by $\mathrm{H}^{+}$. The samples are immediately transferred in the ultra-high vacuum of a molecular beam epitaxy growth chamber (RIBER MBE32) with a base pressure of $\sim 10^{-10}$ Torr. In-situ 
cleaning consisted of a flash annealing at $700^{\circ} \mathrm{C}$ for $30^{\prime}$ in the molecular beam epitaxy chamber, in order to remove any residual native oxide on the UT-SOI surface.

The molecular beam epitaxy reactor is equipped with solid sources (silicon and germanium). We use an electron gun for the deposition of silicon, which consists of a charge containing a silicon ingot, which is heated by electron bombardment vaporizing the $\mathrm{Si}$ atoms. The germanium is source instead, is placed in an effusion Knudsen cell. In this case the evaporation is activated via Joule effect dissipating an electric current in the cell. The growth rates of silicon and germanium for these samples are $\sim 1.2 \mathrm{~nm}$ per minute and $\sim 0.51 \mathrm{~nm}$ per minute respectively. Ge composition from 30 to $100 \%$ and thickness from 10 to $1500 \mathrm{~nm}$ are deposited depending on the final size of the 3D structures obtained via solid state dewetting.

Solid state dewetting was obtained by high temperature annealing. Depending on the initial SiGe layer thickness, Ge content and target morphology (e.g. separated islands, connected structures), the process can be done at temperature ranging between 400 to $800^{\circ} \mathrm{C}$ and time from 30 minutes to 6 hours. Examples of the final morphologies of dewetted structures used as hard masters for nano-imprint lithography are provided in Figure $5{ }^{9}$.

\section{MOLD PREPARATION}

$\mathrm{SiGe}$ is an unusual material for masters that more conventionally are made of $\mathrm{Si}$, metal or plastic. A specific development has been necessary to cast a polymer e.g. (polydimethylsiloxane, PDMS) mold on SiGe for its faithful replication (Figure $1 \mathrm{~b}$ ). Owing to the surface properties of dewetted islands of $\mathrm{SiGe}$ or $\mathrm{Ge}$, an improved hydrophobicity is necessary for demolding. This is obtained only with a much longer aging in $1 \mathrm{H}, 1 \mathrm{H}, 2 \mathrm{H}, 2 \mathrm{H}$-perfluorooctyltriethoxysilane (POTS) vapor with respect to other types of masters used for soft-NIL ${ }^{12,35}$. The same process on a flat Ge surface 
was obtained just after $1 \mathrm{~h}$, pointing to peculiar properties of Ge after dewetting. Indeed, in its crystalline state, germanium, similar to silicon and carbon (diamond), is a covalent solid that crystallizes into a diamond cubic lattice structure. However, when prepared under vacuum conditions, both (100) and (111) surfaces of Ge undergo extensive reconstructions in their surface atomic geometry exhibiting a zwitterionic property ${ }^{36,37}$ : the upper Ge atoms are more nucleophilic, whereas the lower ones are more electrophilic. During dewetting many different crystallographic faces form (according to the equilibrium shape of the material in use). These reconstructions involve the variation of the underlying nucleophilic properties. Thus, in order to enable the silane groups of POTS vapor to react with all nucleophilic sites on the islands surface, the functionalization requires a long time (e.g., about 12 hours in our case, against 1 hour or less, for conventional masters).

PDMS molds were prepared as follows (Figure 1b): firstly, the surface of the master was functionalized with silane group by using $1 \mathrm{H}, 1 \mathrm{H}, 2 \mathrm{H}, 2 \mathrm{H}$-perfluorooctyltriethoxysilane (POTS) in vapor phase for about 12 hours to decrease the surface energy, reducing the interactions with the PDMS chains. A bi-layer hard-PDMS/soft-PDMS stamp was then obtained in two steps. HardPDMS: 1) PP2-RG07 from GELEST kit, part A (base) and part B (for crosslinking) mixed with a molar ratio of 1:10. 2) Pouring PP2-RG07 on the functionalized hard master surface (depending on the master size and morphology the quantity is different) and spin coated: i) Spin speed 300 round-per-minute for 3' '; ii) Spin speed 4000 round-per-minute for 30"'; iii) Curing for 15 min at $65-70^{\circ} \mathrm{C}$ providing a final thickness of $\sim 2000 \mathrm{~nm}$. Soft-PDMS is prepared as follows: i) Mixing the precursors reactants $90 \mathrm{wt} \%$ RTV141A; $10 \mathrm{wt} \%$ RTV141B from BLUESIL; ii) Pouring them atop the hard PDMS. The master with hard and soft-PDMS is cured at $70{ }^{\circ} \mathrm{C}$ for about 12 hours before demolding. 


\section{SOL-GEL DIP-COTING AND NANO-IMPRINT LITHOGRAPHY}

The final metal oxide structures are obtained as follows (Figure 1c). For dense titania $\left(\mathrm{TiO}_{2}\right)$ replicas, the precursor solution was prepared by mixing $1 \mathrm{TiCl}_{4}\left(\mathrm{TiCl}_{4} 99 \%\right.$ SigmaAldrich): 40 EtOH: $12 \mathrm{H}_{2} \mathrm{O}: 5 \times 10^{-4} \mathrm{~F} 127$ (Pluronic F127: polyethylene oxide-polypropylene oxide triblock copolymer (PEO-PPO-PEO, from Aldrich) molar ratios. Dense methylated silica $\left(\mathrm{Si}_{4} \mathrm{O}_{7} \mathrm{Me}_{2}\right)$ was obtained from a solution containing 0.5 TEOS (tetraethylorthosilicate): 0.5 MTEOS (methyltriethoxysilane): 14 EtOH: $5 \mathrm{H}_{2} \mathrm{O}: 0.2 \mathrm{HCl}(1 \mathrm{M}): 1 \times 10^{-4} \mathrm{~F} 127$ molar ratios. After 24 hours of aging, the solutions were dip-coated on glass or on Si substrates at a constant relative humidity (ambient humidity for $\mathrm{SiO}_{2}$ and relative humidity $\mathrm{RH}<10 \%$ for $\mathrm{TiO}_{2}$ ) and with controlled withdrawal speeds between 0.5 and $8 \mathrm{~mm} / \mathrm{s}$ in order to adjust the final thickness ${ }^{38}$. In some cases, the final sol-gel solutions were diluted to obtain the appropriate film thickness.

The PDMS mold was pumped for 10 minutes under primary vacuum and then readily applied (in less than one minute) on the as-deposited sol-gel thin film into a nanoimprint chamber with temperature and relative humidity control ${ }^{35}$. The relative humidity was maintained in the NIL chamber at $70 \%$ for $\mathrm{SiO}_{2}$ and $55 \%$ for $\mathrm{TiO}_{2}$ xerogel films. Before unmolding, the system was cured at $70{ }^{\circ} \mathrm{C}$ for $20 \mathrm{~min}$. The imprinted samples were then annealed at $450{ }^{\circ} \mathrm{C}$ for $10 \mathrm{~min}$.

\section{SPECTROSCOPIC CHARACTERIZATION}

Spectroscopic investigation of the optical properties of the samples in study was performed using 3 different setups. A conventional spectrophotometer (from Perkin Elmer) is used to assess total reflection and diffusion from 250 to $1500 \mathrm{~nm}$. A gonio-reflectometer is used to measure the bidirectional-reflectance distribution function ${ }^{39}$ (BRDF) assessing the structure factor $\mathrm{S}(\mathrm{k})$ of the structures under investigation. We set independently the angle of the incident excitation light beam 
$\left(\theta_{\text {exc }}, \phi_{\text {exc }}\right)$ and that of detection $\left(\theta_{\text {det }}, \phi_{\text {det }}\right) . \theta_{\text {exc }}$ is kept constant during a measurement, whereas the excitation azimuthal angle $\phi_{\text {exc }}$ is always kept fix at 0 degrees in all the measurements. The detection angle $\theta_{\text {det }}$ is scanned from -80 to +80 degrees during a measurement, whereas $\phi_{\text {det }}$ is always kept constant at 0 degrees. For these measurements, the light sources used for excitation are a $630 \mathrm{~nm} \mathrm{CW}$ laser diode and a broad-band tungsten lamp. They are fed in a multimode optical fiber, collimated and shined on the sample as a collimated beam of about $2 \mathrm{~mm}$ in diameter. The scattered light is detected with a multimode optical fiber and detected by a Si-based photodiode (integrating the signal in wavelength, used for the laser excitation) or by a Si-based array (3648 pixels from Oceans Optics, used for measuring the scattering spectra under white light illumination). All the measurements are normalized by a Lambertian reference. We thus measure the BRDF which units are ster. ${ }^{-1}$. An optical microscope is used to excite the sample and collect the reflected light with a high numerical aperture objective lens $(\mathrm{NA}=0.9)$ allowing to integrate over a broad angle of about \pm 64 degrees with respect to the sample normal (see Figure 12a for a sketch of the setup). For these measurements we used a white LED lamp (emitting from $\sim 400$ to $750 \mathrm{~nm}$ ) to illuminate the sample and collecting the light with a multi-mode optical fiber having a diameter of $200 \mathrm{~nm}$ in a confocal geometry. This provides a collection spot on the sample surface of about $5 \mu \mathrm{m}$ in diameter. The light is then fed into a spectrometer and detected by a Si-based array.

\section{ENVIRONMENTAL ELLIPSOMETRY POROSIMETRY}

A characterization of the 2D flat layers was performed in order to determine their properties in terms of refractive index, porosity and roughness. This is done by Environmental Ellipsometry Porosimetry (Figure 2 and 3). The measurements were carried out with a Wollam M200V 
ellipsometer $(350 \mathrm{~nm}-1000 \mathrm{~nm})$ measuring in real time the changes of refractive index of a metal oxide thin layer kept in a chamber with controlled atmosphere where air and water were progressively mixed. We thus measured the refractive index evolution upon adsorption/desorption of water, from which isotherms can be extracted and used to obtain the mesoporosity of the layers (pore volume, pore size distribution, pore interconnection through adapted Kelvin models ${ }^{40,41}$.

\section{EXPERIMENTAL RESULTS}

\section{CHARACTERIZATION OF FLAT METAL-OXIDES LAYERS}

In the case of porous layers (Figure $2 \mathrm{a}$ and d, respectively for porous $\mathrm{TiO}_{2}$ and porous $\mathrm{SiO}_{2}$ ), when the relative solvent pressure increases, adsorption and capillary condensation occur in the pores. The corresponding adsorbed volume fraction of adsorbate is then deduced from the Bruggeman Effective Medium Approximation ${ }^{40}$ (Figure $2 \mathrm{~b}$ and e, respectively for porous $\mathrm{TiO}_{2}$ and porous $\mathrm{SiO}_{2}$ ). The pore size distribution is determined from the isotherm via a modified Kelvin equation to consider the non-spherical geometry of the pores and the thickness of the water layer adsorbed in the pores before capillary condensation (Figure $2 \mathrm{c}$ and $\mathrm{f}$, respectively for porous $\mathrm{TiO}_{2}$ and porous $\left.\mathrm{SiO}_{2}\right)^{40}$. It can be extracted for nanoporous films (up to pores of $\sim 50 \mathrm{~nm}$ in diameter). 

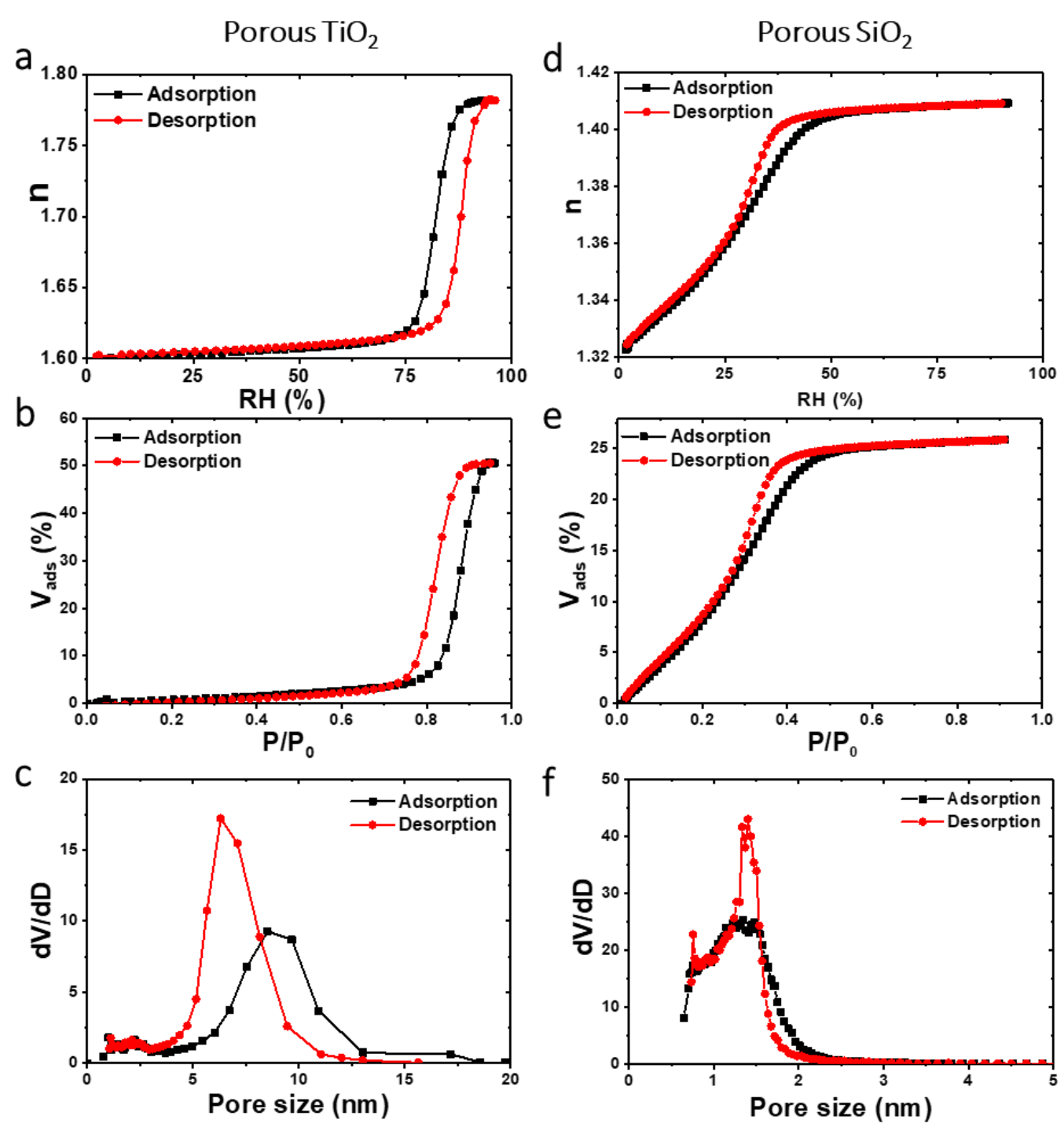

Figure 2. Adsorption/desorption isotherms obtained by ellipsometry porosimetry on a porous $\mathrm{TiO}_{2}$ layer, $100 \mathrm{~nm}$ thick on silicon and on a porous $\mathrm{SiO}_{2}$ layer, $174 \mathrm{~nm}$ thick on silicon. (a) Evolution of the refractive index $\mathrm{n}$ of a porous $\mathrm{TiO}_{2}$ layer a function of the relative humidity $(\mathrm{RH} \%)$ in the atmosphere. (b) Adsorbed water volume (\%) as a function of the water vapor partial pressure for a porous $\mathrm{TiO}_{2}$ layer. (c) Pore size distribution deduced from the adsorption (black) and desorption (red) curves for a porous $\mathrm{TiO}_{2}$ layer. (d) Evolution of the refractive index $\mathrm{n}$ of a porous $\mathrm{SiO}_{2}$ layer a function of the relative humidity ( $\mathrm{RH} \%)$ in the atmosphere. (e) Adsorbed water volume (\%) as a function of the water vapor partial pressure for a porous $\mathrm{SiO}_{2}$ layer. (f) Pore size distribution deduced from the adsorption (black) and desorption (red) curves for a porous $\mathrm{SiO}_{2}$ layer. 
Note that the adsorption and desorption curves do not coincide owing to a well-known poreblocking effect induced by interconnections between pores smaller than the pores themselves (bottleneck pores). Thus, the desorption branch is associated with the interconnections and only the adsorption branch can be exploited to estimate the pore size (even if it occurs out of equilibrium, leading to an overestimation of their dimension). As a first approximation, the adsorption curve provides the size of the smallest dimension of the pores, while the desorption curve provides the size of the interconnections (pore blocking effect) ${ }^{40,41}$

In the case of thin, porous $\mathrm{TiO}_{2}$ layer (Figure $2 \mathrm{a}, \mathrm{b}$ and c) pore and connections size are of the order of $9 \mathrm{~nm}$ and $6 \mathrm{~nm}$ respectively with an overall porosity of about $50 \%$ determining $\mathrm{n} \sim 1.6$. Thus, the case presented here is representative of a rather porous titania layer with a low refractive index. For the thin, porous $\mathrm{SiO}_{2}$ layer (Figure $2 \mathrm{c}, \mathrm{d}$ and e) the porosity is about $25 \%$ of the total volume, the pore diameter and connections are below 1-2 $\mathrm{nm}$. The corresponding refractive index is about 1.32 , thus smaller than the typical value of 1.5 found for dense silica.

A second example of metal oxides layers is provided for dense materials (Figure 3). In these cases, adsorption/desorption isotherms obtained by ellipsometry porosimetry do not provide a precise estimation of the pore size distribution owing to the low variation of refractive index and low pressure at which the pores load with water, preventing the use of Kelvin's law for capillary condensation. However, a measured refractive index of $\sim 2.34$ for dense $\mathrm{TiO}_{2}$ allows to roughly estimate the layer porosity in 10-15\% (Figure 3 a). For dense $\mathrm{SiO}_{2}$ instead the measured refractive index is about 1.43, suggesting a porosity $<10 \%$ vol (Figure $3 \mathrm{~b}$ ). 

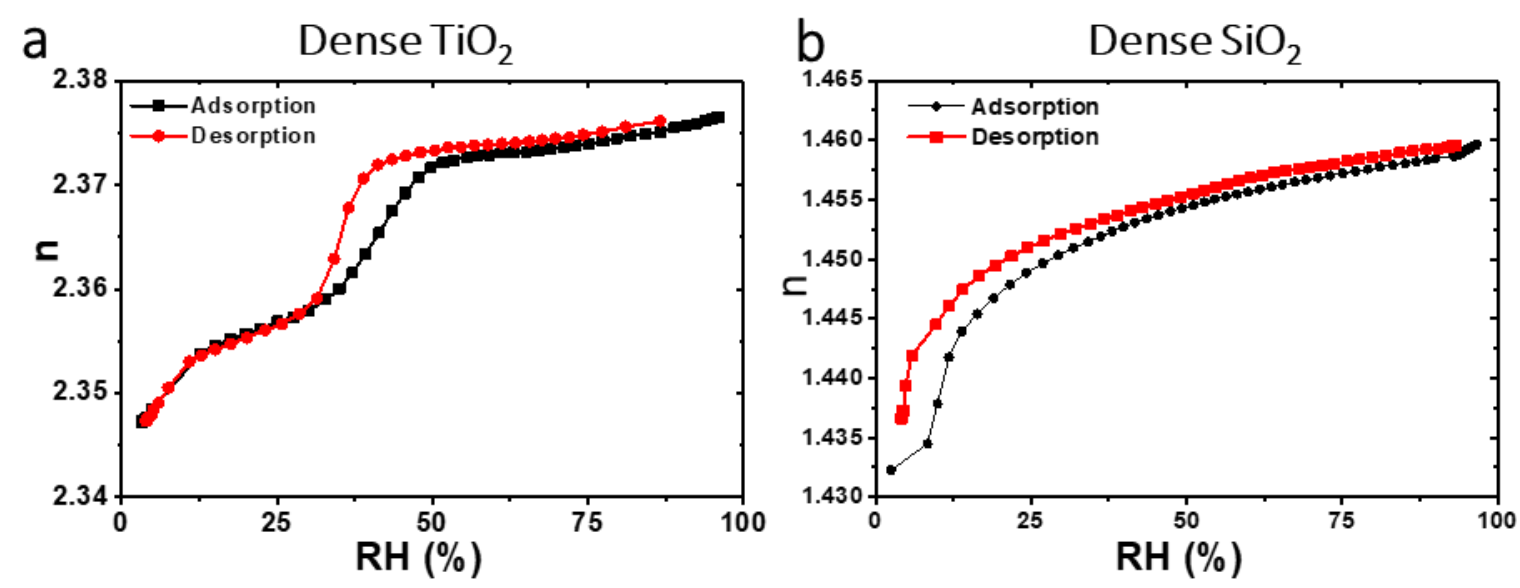

Figure 3. Adsorption/desorption isotherms obtained by ellipsometry porosimetry (a) for a dense $\mathrm{TiO}_{2}$ layer, $62 \mathrm{~nm}$ thick on silicon and (b) for a dense $\mathrm{SiO}_{2}$ layer, $137 \mathrm{~nm}$ thick on silicon.

Further examples of titania layers with different pore size distributions can be found in ${ }^{42,43}$ (D 2-3 nm). For applications in optics, the possibility to adjust the porosity of the sol-gel layers provides a fine tuning of the optical constant of the thin layers and 3D printed structures. It is thus a powerful tool to adjust $\mathrm{n}$ to a desired value (e.g. for anti-reflection coatings ${ }^{12}$ and Bragg mirrors).

As for the case of titania, other examples of silica layers featuring a different porosity can be found in ${ }^{43}$. In this latter case, an average pore size of about $7 \mathrm{~nm}$ is found with a porosity of $37 \%$ determining a refractive index of 1.28 . More generally, a plethora of combinations of porosity, pore size distribution, interconnections between pores can be created at will, providing a full control over the micro- and meso-porosity of metal oxides ${ }^{40}$ and of their refractive index from low ( $\sim 1.1$ for porous $\left.\mathrm{MgF}_{2}{ }^{44}\right)$ to high $(\sim 2.4$ for dense titania). This is for instance possible by playing with the initial concentration of templating agents (e.g. cetyltrimethylammonium tetrachloride, Pluronics F127, and poly(butadiene-b-ethyleneoxide).

\section{ROUGHNESS OF FLAT SOL-GEL LAYERS}



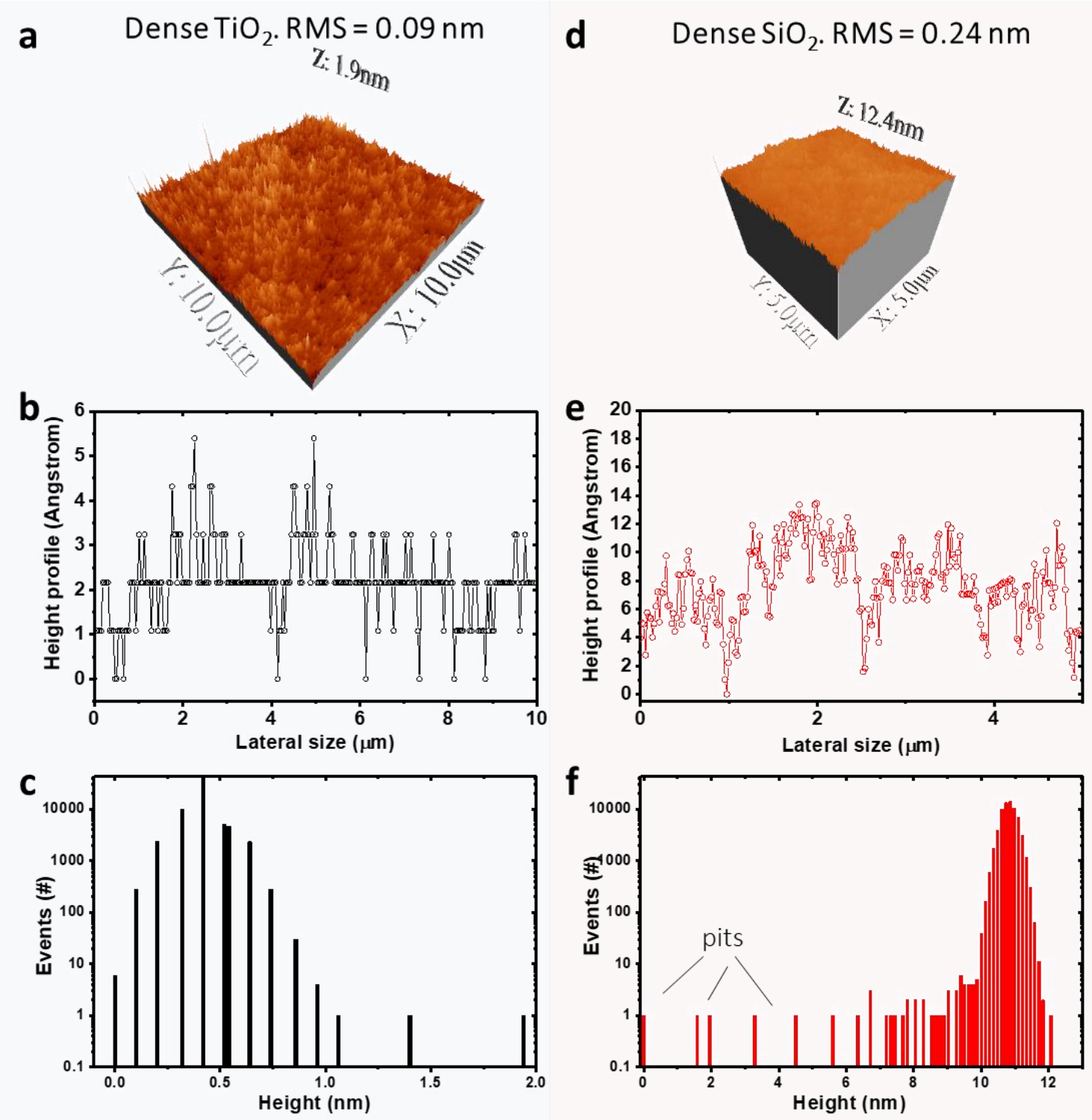

Figure 4. (a) Atomic force microscopy image of a dense $\mathrm{TiO}_{2}$ layer. (b) Height profile deduced from a. (c) Statistical distribution of height deduced from a. (d) Atomic force microscopy image of a dense $\mathrm{SiO}_{2}$ layer. (e) Height profile deduced from d. (f) Statistical distribution of height deduced from d.

Beyond the intrinsic porosity of the sol-gel materials, an important feature is their roughness. In order to address this feature, we measured via atomic force microscopy the height profile of dense $\mathrm{TiO}_{2}$ and $\mathrm{SiO}_{2}$ flat layers (Figure 4). The case of porous layers is not shown. From this characterization we deduce a roughness of about $0.1 \mathrm{~nm}$ for $\mathrm{TiO}_{2}$ and $0.24 \mathrm{~nm}$ for $\mathrm{SiO}_{2}$. In this 
latter case we can observe the presence of a few pits, up to $\sim 10 \mathrm{~nm}$ dip and having a density of about $\sim 5^{*} 10^{6} \mathrm{~cm}^{-2}$. Thus, we conclude that overall, the sol-gel layers are of optical quality. To complement this information, we also mention that from optical spectroscopy measurements, we can address the light scattering of flat layers revealing a diffusion of about $0.4 \%$, as low as that one measured on a polished silicon wafer, which further accounts for the flatness of the sol-gel deposition and lack of scattering centers (see Supplementary Information Figure S.I. 1).

\section{CHARACTERIZATION OF DISORDERED HYPERUNIFORM 3D STRUCTURES BASED ON METAL-OXIDES}
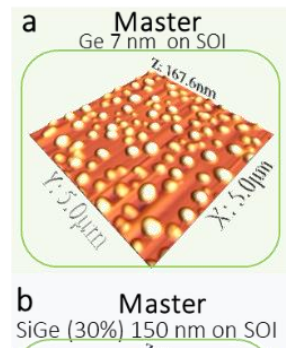

Sige (30\%) $\frac{150 \mathrm{~nm} \text { on } \mathrm{SO}}{2}$
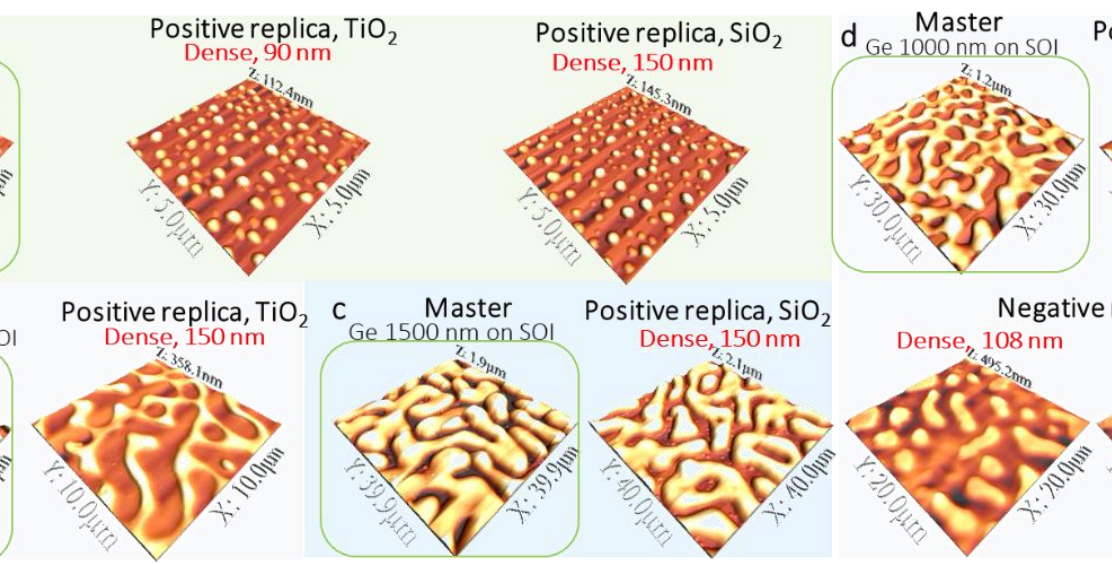

Positive replica, $\mathrm{TiO}_{2}$ Porous $160 \mathrm{~nm}$
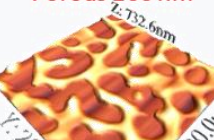

- 12

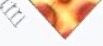

Negative replica, $\mathrm{TiO}_{2}$
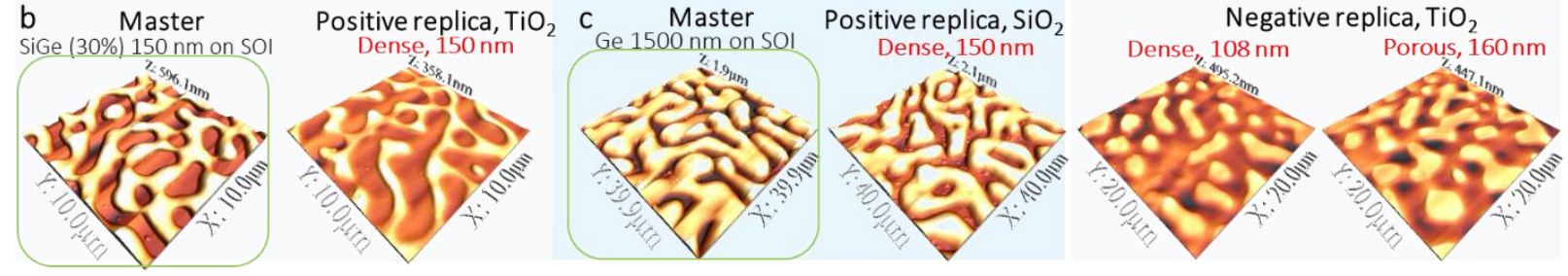

Figure 5. (a) $\mathrm{SiGe}$ master with isolated islands (left panel) and positive replicas in dense $\mathrm{TiO}_{2}$ and $\mathrm{dense}_{\mathrm{SiO}}$ (respectively central and right panels). (b) SiGe master of connected structures (left panel) and positive replica in dense $\mathrm{TiO}_{2}$ (right panel). (c) $\mathrm{SiGe}$ master of connected structures (left panel) and positive replica in dense $\mathrm{SiO} 2(\mathrm{right}$ panel). (d) SiGe master of connected structures (top left panel) and positive replica in porous $\mathrm{TiO}_{2}$ (top right panel), negative replica in dense $\mathrm{TiO}_{2}$ (bottom left panel) and negative replica in porous $\mathrm{TiO}_{2}$ (bottom right panel).

Starting from plain, as-prepared, sol-gel coatings we can replicate all the different morphologies (e.g. islands, connected structures, Figure 5). At a glance, we show the hard SiGe masters obtained via solid state dewetting and the corresponding $\mathrm{TiO}_{2}$ and $\mathrm{SiO}_{2}$ replicas of small 
disconnected islands for dense materials (Figure 5 a) and connected structures for dense materials (Figure $5 \mathrm{~b}$ and $\mathrm{c}$ ). These metal oxides-based nano- and micro-architectures can be printed on glass and on silicon ${ }^{12,42,43}$ and thus, all combinations of morphology, material, size and substrate are in principle possible.

\section{INVERTED STRUCTURES: NEGATIVE REPLICAS}

As a further example of the versatility of our method, we showcase the possibility to invert the original structures by printing their negative replica. Inverting a mold allows to limit the use (and deterioration) of the original hard master, increasing its lifetime. Moreover, some designs might be very complex or time-consuming to obtain by lithography, whereas their inverse is not.

A simple way to invert a mold is summarized in Supplementary Information Figure S.I. 2. If liquid PDMS before (reticulation) is directly poured on a PDMS mold and cured, cross-linking occurs at the interface and demolding is impossible. In fact, PDMS is never fully crosslinked and even after long annealing time some functions can still be reactive. In order to easily separate negative from positive PDMS, different techniques were developed to change the surface functionalization of PDMS ${ }^{45-47}$ These techniques are hardly exploitable for small structures (e.g. of sub-micron dimension), in particular when densely packed. Thus, we added a thin conformal $\mathrm{SiO}_{2}$ layer of few tens of nanometers deposited via plasma enhanced chemical vapor deposition. Positive and negative molds, made using $15 \mathrm{~nm}$ of $\mathrm{SiO}_{2}$ as buffer layer, were then used for imprinting metal oxides.

To confirm the good quality of the inverted molds, the replication of two different kinds of ordered patterns were evaluated: a network of densely packed dots (depth $100 \mathrm{~nm}$, diameter 270 $\mathrm{nm}$, pitch $700 \mathrm{~nm}$ ) and large rings (depth $400 \mathrm{~nm}$, outer diameter $D_{\text {ext }}=6 \mu \mathrm{m}$, inner diameter $D_{\text {int }}$ 
$=3 \mu \mathrm{m}$ and pitch $10 \mu \mathrm{m})$. The masters and resulting positive/negative replicas were thus analyzed by AFM in order to compare their dimensions. AFM images are shown in the Supplementary Information Figure S.I. 2, 3 and summarized in the corresponding Supplementary Information Table S.I. 1 for the dense $\mathrm{TiO}_{2}$ case.

This technique is used here to replicate and invert the complex features framed by spontaneous dewetting to obtain more diverse structures from the same master. Positive and negative molds were fabricated by following the protocol detailed above on various sol-gel formulations (Figure 5 c) such as dense $\mathrm{TiO}_{2}$, porous $\mathrm{TiO}_{2}$ and porous $\mathrm{SiO}_{2}$ (not shown) producing positive and negative replicas of the dewetted structures.

\section{DISORDERED HYPERUNIFORMITY OF METAL OXIDE STRUCTURES}

In what follows we will address the features of characterizing the disorder of the master and replicas. We will focus only on dense $\mathrm{TiO}_{2}$ as it is a more interesting material for applications in photonics (e.g. provided its larger refractive index with respect to $\mathrm{SiO}_{2}$ ). However, as shown in Figure 5, the replication of the same structures in $\mathrm{SiO}_{2}$ and in porous materials is straightforward. 

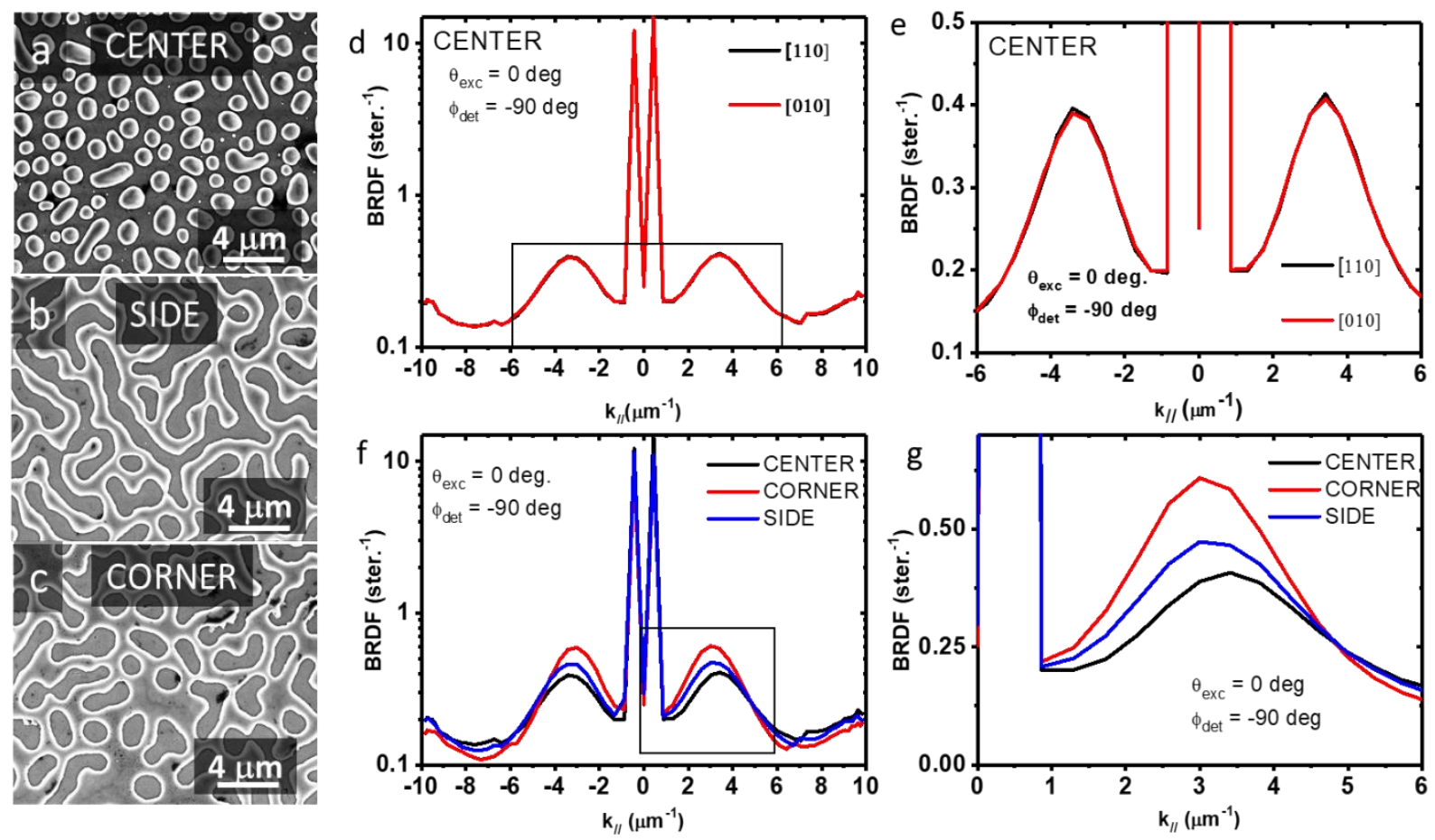

Figure 6. (a) Scanning electron micrograph (SEM) of a master (150 nm SiGe after dewetting) at its center. (b) SEM of the master at the edge. (c) SEM of the master at the corner. (d) Bidirectional reflectance distribution function (BRDF) of the master measured at its center for fixed excitation at normal incidence for two orthogonal crystallographic directions. (e) Blow-up of (d). (f) Bidirectional reflectance distribution function (BRDF) of the master measured at its center, its side and its corner for fixed excitation at normal incidence. (g) Blow-up of (f).

We performed a spectroscopic investigation of the $\mathrm{dHU}$ character of a SiGe master and its metal oxide replica, addressing their structure factor $\mathrm{S}(\mathrm{k} / /)$ via light scattering in the far field (where $\mathrm{k} / /$ $=2 \pi / \lambda * \sin \theta_{\text {det }}$ is the in-plane wavevector, $\lambda$ is the wavelength of the incident light and $\theta_{\text {det }}$ is the detection polar angle with respect to the sample normal).

We first characterize the BRDF of a SiGe-based master obtained by spinodal solid-state dewetting of $150 \mathrm{~nm}$ of $\mathrm{Si}_{0.7} \mathrm{Ge}_{0.3}$ on ultra-thin silicon on insulator ${ }^{9}$ (Figure 2). The far-field scattering of the laser shined at normal incidence $\left(\theta_{\mathrm{exc}}=0\right.$ degrees $)$ at the center of the sample (where the morphology of the dewetted structures displays disconnected islands, Figure $6 \mathrm{a}, \mathrm{d}$ and 
f), shows an intense peak at $\mathrm{k}_{/ /}=0 \mu \mathrm{m}^{-1}\left(\theta_{\mathrm{det}}=0\right.$ degrees), a sharp decrease and two symmetric lobes at about $\mathrm{k} / / \sim \pm 3.4 \mu \mathrm{m}^{-1}$. For the sake of thoroughness, we mention that the deep in the specular reflection at $\mathrm{k} / /=0 \mu \mathrm{m}^{-1}$ degrees is due to a shadow effect of the detecting arm passing in front of the excitation arm. It is just an artificial effect. Measuring $S(k /)$ in two orthogonal directions ([110] and [010], in-plane crystallographic directions of the (001) surface of the original SOI, by rotating the sample of 90 degrees), provides identical results within experimental error accounting for the uniformity of the scattering that is configured as a ring in the far-field (Figure $6 \mathrm{~d}$ and f).

Generally speaking the degree of hyperuniformity of a disordered material can be quantified by extrapolating the value of $\mathrm{S}(\mathrm{k}=0)$. This quantity is strictly zero for ideal $\mathrm{dHU}$ materials whereas in all experimental cases it always features a finite value. The smaller the value, the higher the degree of hyperuniformity ${ }^{48}$. Very often, accessing the real value of $\mathrm{S}(\mathrm{k}=0)$ can be challenging and depending on the experimental tools and method analysis overestimations are likely possible. The laser scattering method used here does not allows to explore very small $\mathrm{k}$, owing to a limited angular resolution ( $\sim 1$ degree) and the presence of a very intense specular reflection peak at $\mathrm{k}=0$. Thus, we can extrapolate an $\mathrm{S}(\mathrm{k}=0) \sim 0.15$ which is not far from typical values found for spontaneous dHU patterns ${ }^{48-50}$. From this analysis it emerges an effective hyperuniform character of the masters in use as expected for these kinds of patterns ${ }^{30}$ and from the analysis of the spectral density measured from large atomic force microscopy images in similar samples ${ }^{9}$.

It is interesting to note that $\mathrm{S}(\mathrm{k} / /)$ is sensitive to the different morphologies of the underlying structures. Owing to gradients in the annealing temperature of the sample in the molecular beam epitaxy machine, the dewetting at the center and at the edges evolves at a slightly different speed, 
resulting in different morphologies, ranging from holes (Figure $6 \mathrm{c}$ ), to connected structures (Figure $6 \mathrm{~b}$ ), to islands (Figure 6 a). Measuring $\mathrm{S}(\mathrm{k} / /)$ in these three areas is possible to follow its evolution (Figure $6 \mathrm{e}$ and $\mathrm{g}$ ) with the maximum moving from about 3 to $3.4 \mu \mathrm{m}^{-1}$. The deep at small wavevectors is more pronounced for connected structures with respect to the islands, in agreement with the previous assessment of the dHU character of this kind of samples ${ }^{9}$.

A similar measurement is repeated by changing the angle of incidence of the excitation from $\theta_{\mathrm{exc}}=0$ to -40 degrees in steps of 10 degrees (Supplementary Information Figure S.I. 4). Also, in this case the scattering is redistributed in a ring centered around the specular reflection

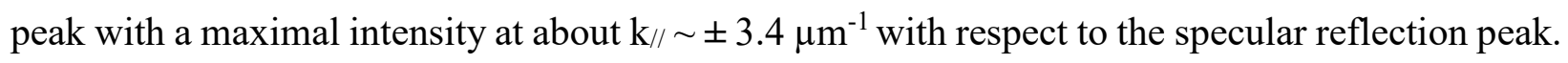
Thus, the scattering ring in the far-field shifts according to the incidence angle of the excitation laser, as expected for this kind of disordered structures $\left(\mathrm{I}_{\mathrm{ring}}\left(\mathrm{k}_{/}\right)=\mathrm{S}\left(\mathrm{k}_{/ /}-\mathrm{k}_{\text {in }}\right)\right.$ where $\mathrm{k}_{\text {in }}$ is the projection on the plane of the sample of the wavevector of the incident radiation ${ }^{22}$. The same measurement performed on a titania-based replica of the master shows exactly the same behavior with the ring at $\mathrm{k} / / \sim \pm 3.4 \mu \mathrm{m}^{-1}$ degrees and an overall lower scattering intensity (Figure 7 a). This latter feature is explained by the lower refractive index of the titania used for soft-NIL ( $n \sim 2.2)$ with respect to the SiGe master ( $\mathrm{n} \sim 4$ ). Thus, beyond the structural characterization performed with the AFM that can provide only local information ${ }^{9}$, the use of the BRDF allows us to average the properties over a larger scale (e.g., in our case several $\mathrm{mm}^{2}$ ) accounting for the similar $\mathrm{dHU}$ features of master and replicas. 

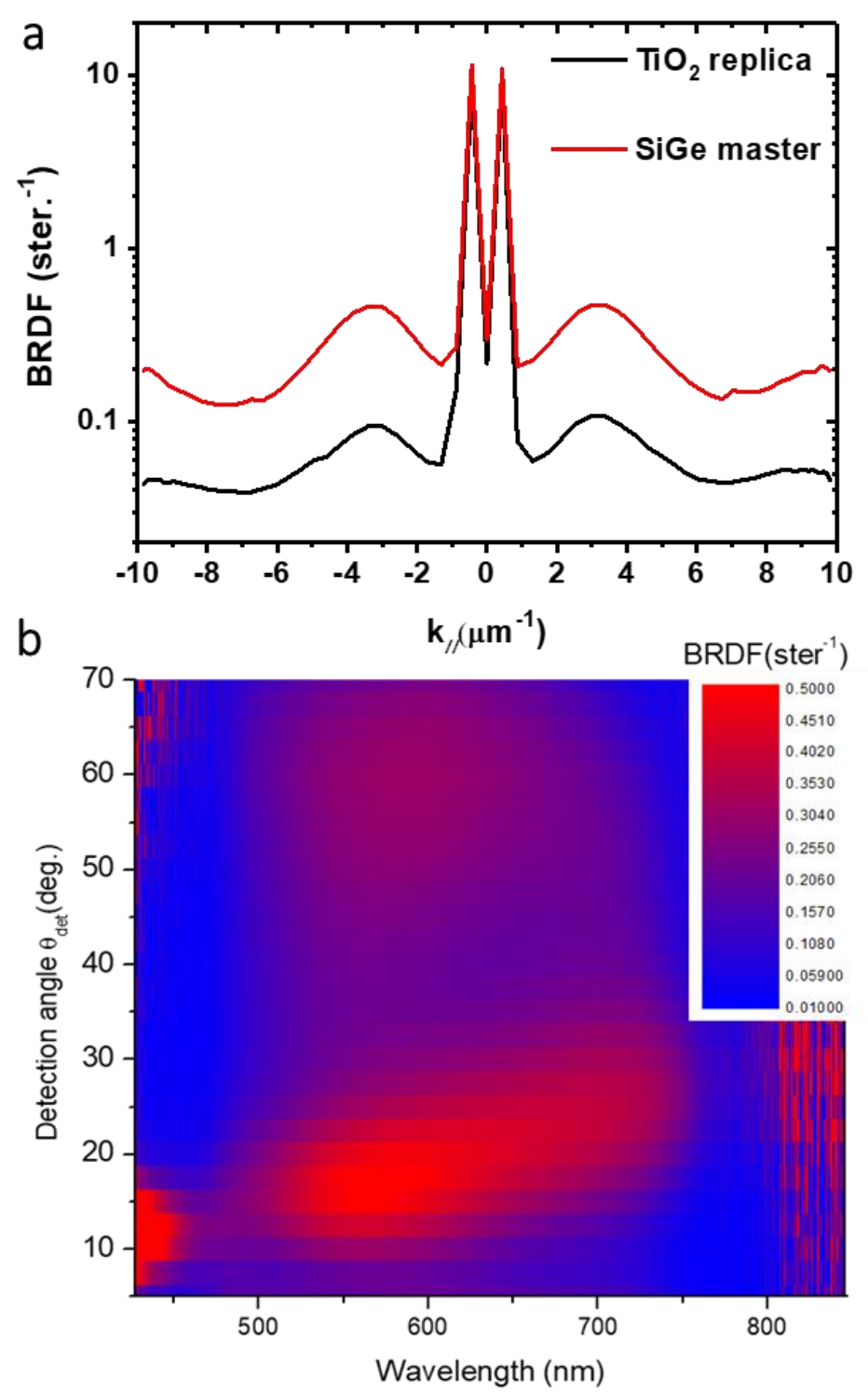

Figure 7. (a) BRDF for the SiGe master (dewetted $150 \mathrm{~nm} \mathrm{SiGe)}$ and for the titania replica. Both measurements are taken at the sample center. (b) Color-intensity map of spectrally-resolved $\mathrm{BRDF}$ for the $\mathrm{TiO}_{2}$ replica on silicon under white light illumination.

A final assessment of the dHU character is provided by measuring the BRDF by using white light and spectrally resolving the measurement (Figure $7 \mathrm{~b}$ ). As expected from the presence of a ring at a defined in-plane wavevector in $\mathrm{S}(\mathrm{k} / /)$, the far-field scattering measured by the BRDF as a function of the incident wavelength shows a linear trend of the angle at which this ring is found 
$\left(\mathrm{k}_{/ / \text {ring }}=2 \pi / \lambda^{*} \sin \theta_{\max }\right)$. This latter result mimics what was found in reference ${ }^{51}$ by M. CastroLopez and coworkers for a dHU metasurface obtained via top-down lithography of a gold layer. We also note that similar measurements performed on dewetted samples made of SiGe, but obtained in completely different growth conditions did not provide the same kind of results in terms of BRDF ${ }^{52}$ (no ring was observed) confirming that the dHU features shown here are specific of spinodal-like dewetting.

\section{SHRINKING AND RESIDUAL LAYER OF PRINTED DISORDERED HYPERUNIFORM}

\section{STRUCTURES}
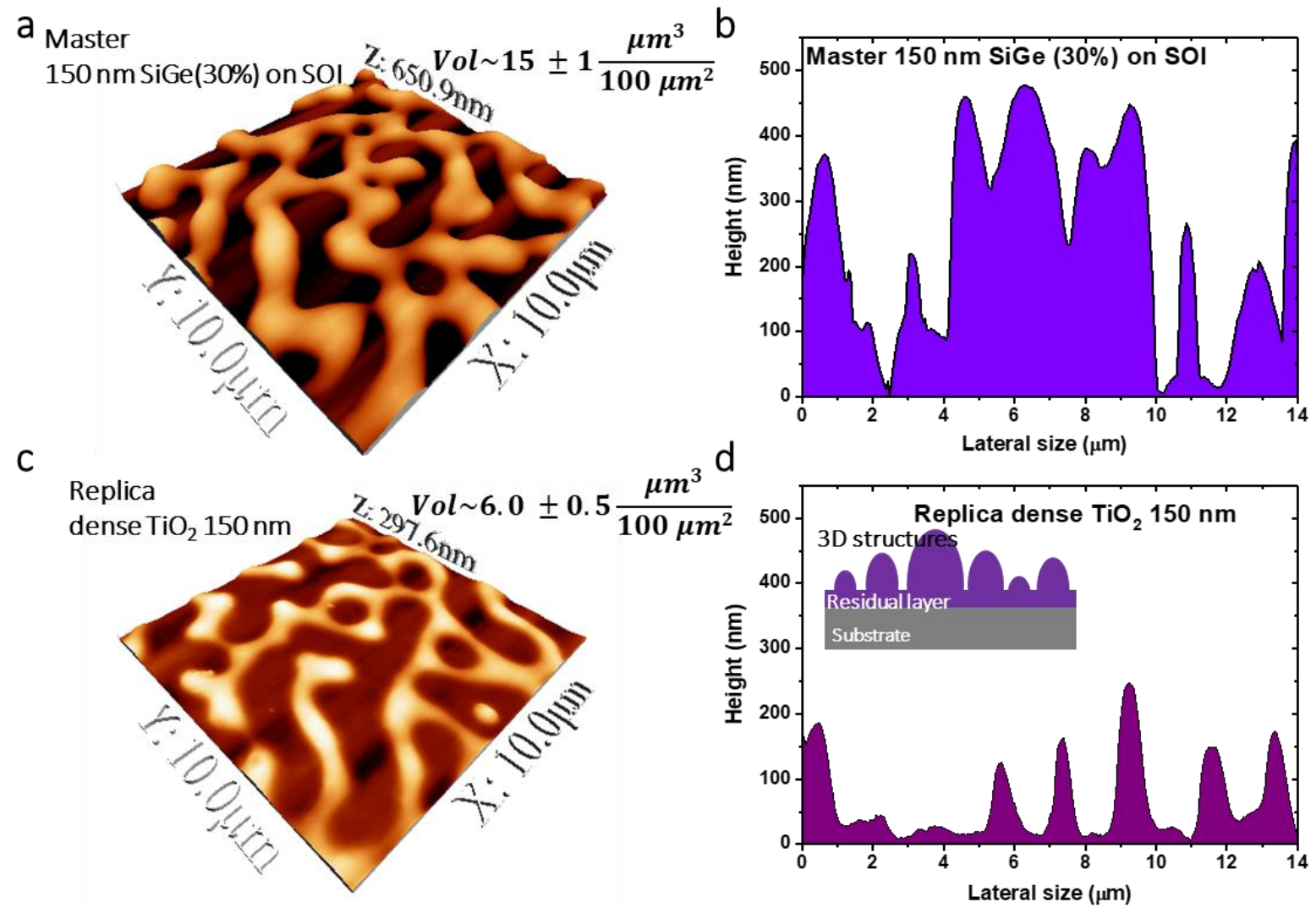

Figure 8. (a) Atomic force microscopy image of a SiGe master bearing connected structures. (b) Height profile extracted from (a). (c) Atomic force microscopy image of a dense $\mathrm{TiO}_{2}$ replica. (d) Height profile extracted from (c). The inset shows a sketch of the printed structures sitting atop of flat residual layer. 
Although to a first look, the metal oxide replicas correspond well to the master morphologies, a more precise assessment of their features shows that for dense $\mathrm{TiO}_{2}$ a relevant size shrinking occurs (Figure 8). We use atomic force microscopy to measure the overall volume of the $3 \mathrm{D}$, dewetted SiGe structures (obtained from a $150 \mathrm{~nm}$ thick SiGe layer) resulting in $\sim 15 \mu \mathrm{m}^{3}$ on a surface of $100 \mu \mathrm{m}^{2}$ and maximal height of about $400 \mathrm{~nm}$ (Figure $8 \mathrm{a}$ and $\mathrm{b}$ ). By using an equivalent volume of dense titania (evaluated by ellipsometry after calcination) for the replica, we find a total volume of $\sim 6 \mu \mathrm{m}^{3}$ on a surface of $100 \mu \mathrm{m}^{2}$ and a maximal height of $\sim 200 \mathrm{~nm}$ (Figure $8 \mathrm{a}$ and b).
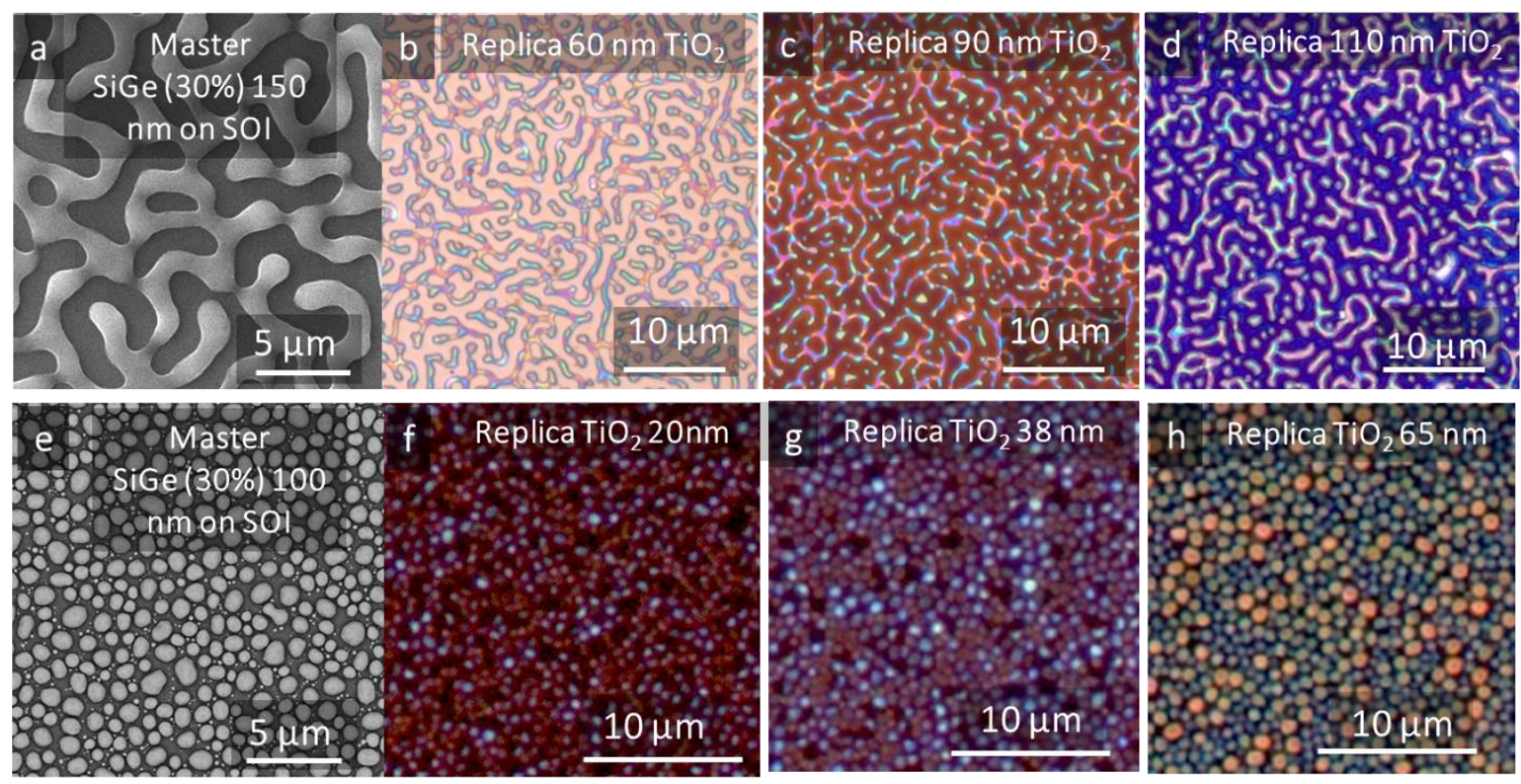

Figure 9. (a) Scanning electron microscope image of the hard SiGe master bearing connected structures. Optical microscope image (bright field, 100X magnification, numerical aperture NA=0.9) for a (b) $60 \mathrm{~nm}$ (c) $90 \mathrm{~nm}$ (d) 110 nm thick, dense $\mathrm{TiO}_{2}$ replica. (e) Scanning electron microscope image of the hard $\mathrm{SiGe}$ master bearing isolated islands. Optical microscope image (dark field, 100X magnification, numerical aperture NA $=0.9)(\mathbf{f})$ for a $20 \mathrm{~nm}(\mathrm{~g})$ for a 38 nm (h) for a $65 \mathrm{~nm}$ thick, dense $\mathrm{TiO}_{2}$ replica.

This observation clearly points out that the printed structures are shrink with respect to those on the original master and that they sit atop a flat residual layer (see the inset of Figure $8 \mathrm{~d}$ ). 
A further indirect evidence of these features can be observed by optical inspection of the printed structures (Figure 9). When changing the thickness of the dense $\mathrm{TiO}_{2}$ used for printing, the reflected colors from both 3D structures and from the flat areas nearby, abruptly changes as revealed by bright-filed, optical microscope images (Figure 9 b-d). This can be explained by constructive and destructive interferences in the residual layer as well as light scattering from the 3D structures acting ad Mie resonators as visible in dark-field optical microscope images (Figure $9 \mathrm{f}$-h). As shown later, these morphological features have a deep influence on the optical properties of the replicas.
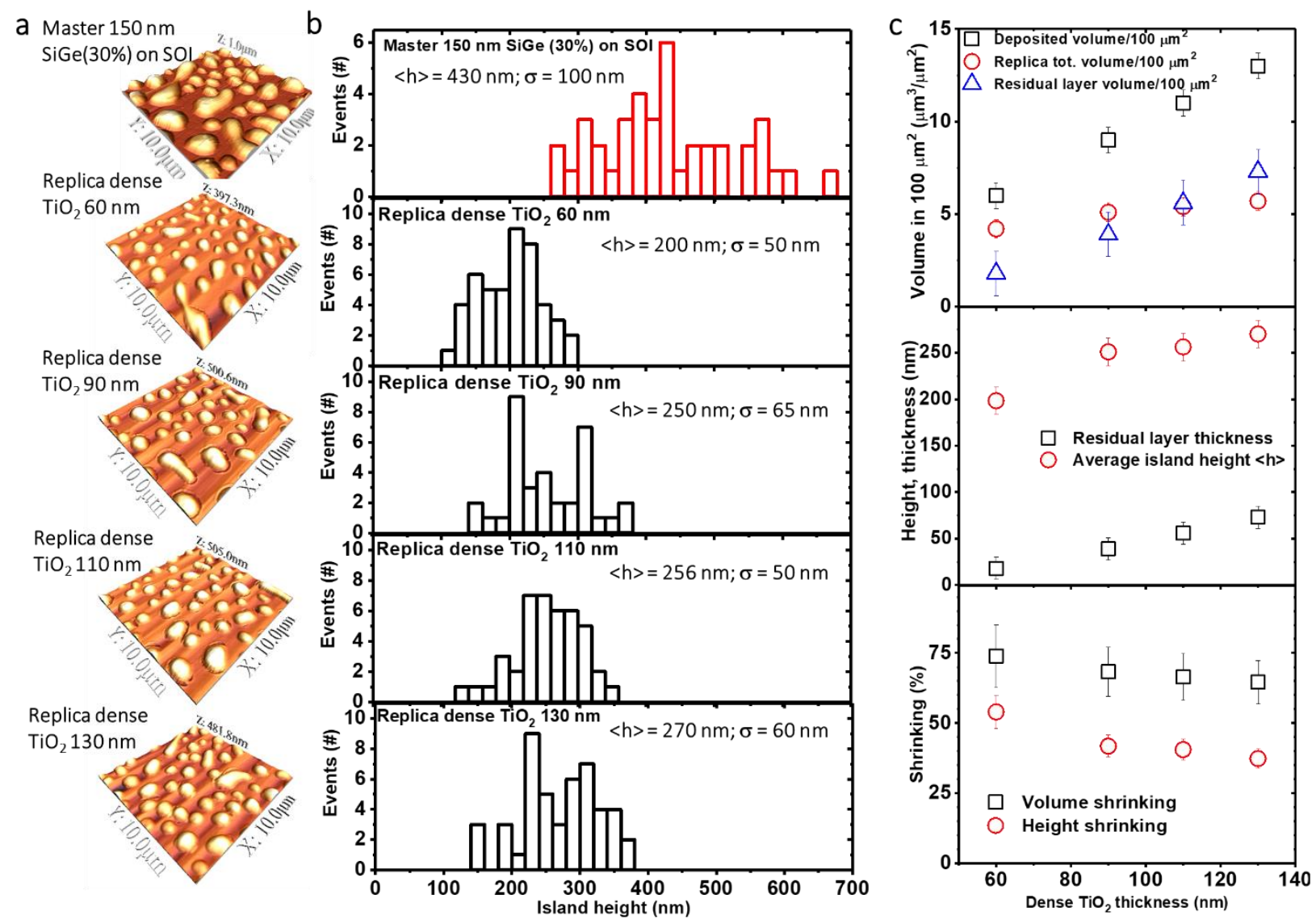

Figure 10. (a) From the top to the bottom panel: AFM images of a SiGe master bearing isolated islands, dense $\mathrm{TiO}_{2}$ replica printed on a $60 \mathrm{~nm}, 90,110$ and $130 \mathrm{~nm}$ thick layers. (b) Statistical distribution of islands height as deduced from the atomic force microscope images in a. On each panel the average height $<\mathrm{h}>$ and corresponding standard 
deviation $\sigma$ are reported. (c) Top panel: deposited volume (squares), total volume of the printed structures (circles) and residual layer volume (triangles) as a function of the deposited $\mathrm{TiO}_{2}$ thickness. Central panel: average islands height $<\mathrm{h}>$ (circles) and residual layer thickness (squares). Bottom panel: height (circles) and volume shrinking of the replicas with respect to the master as a function of the deposited layer thickness. Other examples of this analysis are provided in the Supplementary Information Figure S.I. 5 and 6.

A more quantitative and systematic investigation of shrinking and residual layer thickness can be performed for samples bearing separate islands (Figure 10). By measuring the island height (Figure $10 \mathrm{~b}$ ), their total volume (Figure $10 \mathrm{c}$, top panel) and knowing the thickness of the deposited material, we estimate the thickness of the residual layer (Figure $10 \mathrm{c}$, central panel), height and volume shrinking with respect to the original structures on the master (Figure $10 \mathrm{c}$, bottom panel). From this investigation (and from other reported in the Supplementary Information Figure S.I. 5 and 6) we can see a sub-linear increase of the islands height and a faster (linear) increase of the residual layer as a function of the deposited layer thickness. Moreover, the 3D structures display a relevant shrinking both in height (55-40\%) and in volume (75-60\%). Although the shrinking is reduced for thicker layers, the tendency is very weak.

\section{OPTICAL PROPERTIES OF DISORDERED HYPERUNIFORM PRINTED STRUCTURES}

As a possible application we provide a characterization of the optical response under white light illumination of dense $\mathrm{TiO}_{2}$-based structures, showing an anti-reflective coating (ARC) character when printed on bulk Si. Part of the samples used as masters have been already characterized in reference ${ }^{9}$. For optical applications we produced series of ad-hoc SiGe and Ge islands in order to optimize their size and shape for as anti-reflection coatings at visible and near-infrared frequencies. 
Light reflection and diffusion are measured from near-UV up to near-IR at quasi-normal incidence (11 degrees with respect to the sample normal) with a spectrophotometer mounting an integrating sphere. We showcase four different examples of $\mathrm{TiO}_{2}$ samples having an initial sol-gel layer thickness of 70, 95, 120 and $147 \mathrm{~nm}$ deposited on bulk Si (Figure 11), all obtained starting from a $100 \mathrm{~nm}$ dewetted Ge master.
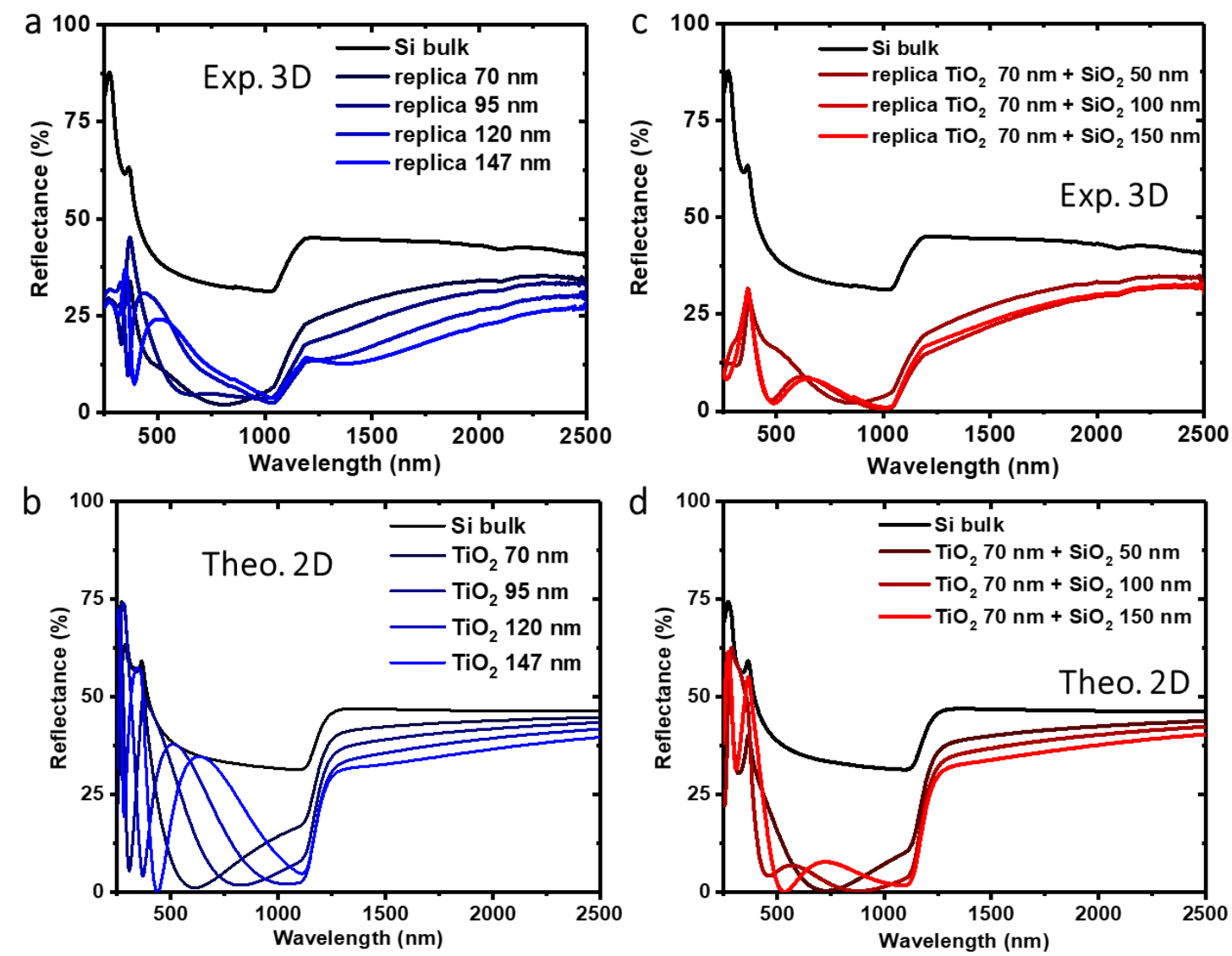

Figure 11. (a) Reflectance measurements for bare bulk Si, and 3D disordered hyperuniform replicas made of dense $\mathrm{TiO}_{2}$ on Si printed on 70, 95, 120 and $147 \mathrm{~nm}$ thick layers. Atomic force microscope images of 70, 95120 and 147 $\mathrm{nm}$ samples are provided in the Supplementary Information Figure S.I. 5 (a). (b) Theoretical simulations of reflectance of $\mathrm{Si}$ bulk and flat layers of $\mathrm{TiO}_{2}$ on $\mathrm{Si}$ having a refractive index of 2.2 (similar to that of the $3 \mathrm{D}$ printed structures) and thickness of 70, 95, 120 and $147 \mathrm{~nm}$ (as those used for nanoimprint in a). (c) Reflectance measurements for bare bulk $\mathrm{Si}$ and 3D disordered hyperuniform replicas made of dense $\mathrm{TiO}_{2}$ on $\mathrm{Si}$ printed on $70 \mathrm{~nm}$ of $\mathrm{TiO}_{2}$ on $\mathrm{Si}$ and covered 
with additional 50, 100 and $150 \mathrm{~nm}$ of dense $\mathrm{SiO}_{2}$ having a refractive index of 1.35. Atomic force microscope images of the $70 \mathrm{~nm}$ replica covered with 50 and $100 \mathrm{~nm}$ of $\mathrm{SiO}_{2}$ are shown in samples are provided $\mathrm{n}$ the Supplementary Information Figure S.I. 7. (d) Theoretical simulations of reflectance of $\mathrm{Si}$ bulk and flat layers of dense $\mathrm{TiO}_{2}$ having a refractive index of 2.3 (similar to that of the 3D printed structures), thickness of $70 \mathrm{~nm}$ (as the deposited layer used for nanoimprint in a) and covered with a flat layer of dense $\mathrm{SiO}_{2}$ having a refractive index of 1.43 (as those used in b). Theoretical simulations of light reflection for different incidence angles of a bilayer of $\mathrm{TiO}_{2} 70 \mathrm{~nm}$ topped with $150 \mathrm{~nm}$ oaf $\mathrm{SiO}_{2}$ are shown in the Supplementary Information S.I. 7.

All the samples show a remarkable reduction of the reflected intensity with respect to the bare bulk $\mathrm{Si}$ in a broad spectral range, extending from visible to near-infrared. Note that the presence of a sharp increase in the reflected light intensity at about $1150 \mathrm{~nm}$ is due to the transparency of Si below the energy bandgap. Thus, at near-infrared frequency, the reflection spectrum is determined also by the light reflected at the bottom surface of the wafer. Overall, the ARC effect shifts to longer wavelengths when increasing the thickness of the printed $\mathrm{TiO}_{2}$ layers. This behavior can be explained as the combination of two effects: 1) the larger size of the 3D printed objects that act as Mie resonators ${ }^{43}$ shifting the resonant light coupling into the substrate to longer wavelength ${ }^{52,53}$ and 2) the increase of the $2 \mathrm{D}$ residual layer thickness underneath the $3 \mathrm{D}$ objects that produces etaloning with a first reflection minimum at progressively longer wavelength.

At some wavelength the reflected intensity can be as small as $2 \%\left(70 \mathrm{~nm} \mathrm{TiO}_{2}\right.$ printed structures, Figure 11a) and below 5\% in broad interval of wavelength (e.g. about a $400 \mathrm{~nm}$ for $120 \mathrm{~nm} \mathrm{TiO}_{2}$ printed structures, Figure 11a). The 2D flat layers counterpart can be simulated by transfer matrix formalism based on the Fresnel equation (Figure 11b). If compared to this latter case, the 3D printed structures show better performances as ARC. In fact, although the 2D layers can exhibit minima of reflection well below $0.05 \%$ (e.g. at see the case of flat $\mathrm{TiO}_{2}$ at $\sim 440 \mathrm{~nm}$, Figure $11 \mathrm{~b}$ ), these low values are limited to a very narrow spectral band-width. 
Further insight on the optical properties of 3D printed structures can be obtained by detecting only on the scattered light (removing the specular reflection from the spectra). Diffusion ${ }^{42,43,54}$ plays an important role in limiting the ARC performances of the titania-based soft-NIL structures (not shown). Thus, to limit the scattering and improve the ARC effect, we further processed the samples by adding via dip-coating a $\mathrm{SiO}_{2}$ layer with a variable thickness ranging from 50 to 150 $\mathrm{nm}$ atop the $70 \mathrm{~nm} \mathrm{TiO}_{2}$ structures (Figure 11c). We observe a further reduction in the reflected intensity explained by two effects: 1$)$ a smoother change of refractive index from air $(n=1)$, to $\mathrm{SiO}_{2}(\mathrm{n} \sim 1.35)$, to $\mathrm{TiO}_{2}(\mathrm{n} \sim 2.2)$, and to $\mathrm{Si}(\mathrm{n} \sim 4)$, and 2$)$ a neat reduction of light scattering from the $\mathrm{TiO}_{2}$ structures as confirmed by diffusion measurements (not shown). As found in the previous case, overall, the performances of $3 \mathrm{D}$ structures topped by $\mathrm{SiO}_{2}$ are superior to the flat $2 \mathrm{D}$ layers counterpart (Figure 11c).

3D ARC can couple the light in the underlying substrate also when changing the incident angle of light, a feature not matched by conventional stacks of 2D layers. In order to assess this property, we compare the reflection of 3D structures with the flat 2D layers by exciting the sample and collecting the reflected light with a high numerical aperture objective lens $(\mathrm{NA}=0.9)$ allowing to integrate over an angle of about \pm 64 degrees with respect to the sample normal (Figure 12a). These spectra were normalized to that one detected on a bare Si substrate (thus a reflection intensity of 1 corresponds to Si reflection).

Exploiting the lateral resolution of the confocal microscope, we can test the homogeneity of the reflection spectra in different parts of the sample (Figure 12.b). Fluctuation below $5 \%$ are found form one point to the other, accounting for the uniform ARC properties across the sample. Furthermore, comparing the reflection spectra from the printed areas with those collected in the 
flat parts (Figure 12c and d), we can observe again a superior performance of the former case with respect to the latter as expected for 3D ARC.

a

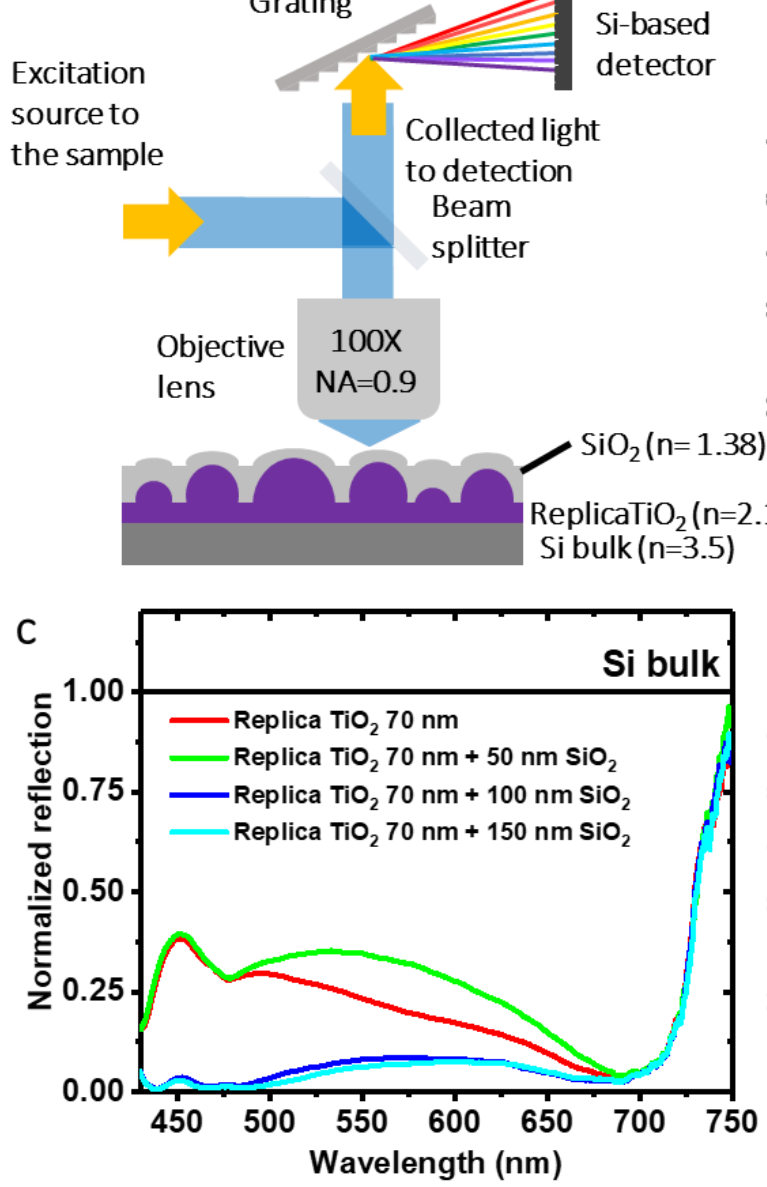

b

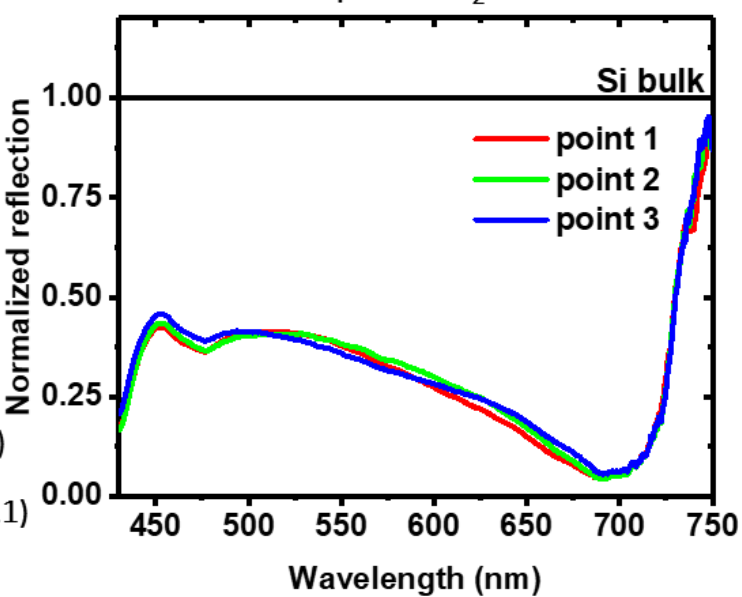

Replica $\mathrm{TiO}_{2} 70 \mathrm{~nm}$

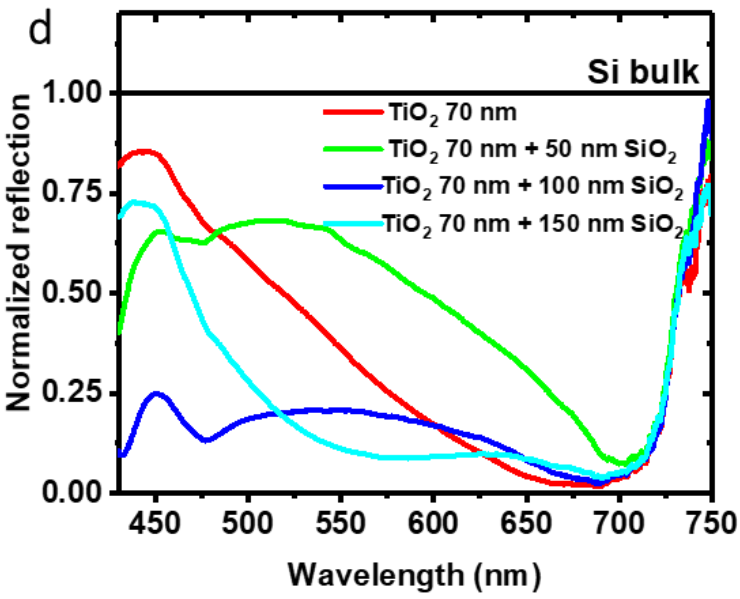

Figure 12. (a) Sketch of the sample and the optical setup for light illumination and detection. We use a LED light source coupled into a high numerical aperture objective lens $(\mathrm{NA}=0.9)$, determining an excitation/collection angle of \pm 64 degrees with respect to the sample normal. The light is dispersed by a grating and detected by a Si based CCD array. (b) Normalized reflection of a 3D replica on $70 \mathrm{~nm}$ thick $\mathrm{TiO}_{2}$ detected in several parts of the sample spaced by hundreds of $\mu \mathrm{m}$ one from the other. (c) Normalized reflection of 3D replicas on $70 \mathrm{~nm}$ thick $\mathrm{TiO}_{2}$ for the bare case and for the $\mathrm{SiO}_{2}$ topped cases. (d) Normalized reflection of 2D layers for $70 \mathrm{~nm}$ thick $\mathrm{TiO}_{2}$ and for the $\mathrm{SiO}_{2}$ topped cases. 
Thus, in spite of the good properties of stacks of 2D layers at certain wavelength, the 3D counterpart can overperform this classic approach being both broad-band and broad angle. It is in fact well known that 2D layers can be optimized for a limited set of incidence angles (see also the Supplementary Information Figure S.I. 8 for simulations of stacks of 2D layers as a function of incidence angle).

\section{DISCUSSION}

The advantage of our fabrication protocol over previous reports ${ }^{13,14,16-20,22}$ of $\mathrm{dHU}$ devices is manifold. Most of the techniques used for photonic applications at visible and near-infrared frequencies exploit top-down methods (e.g., e-beam lithography and reactive ion etching). As such, they are hardly scalable, limiting the range of applicability of dHU materials. Self-assembly for dHU system ${ }^{23-31,55}$ can be typically implemented in liquid and soft materials, featuring a low dielectric constant (low refractive index) less appropriate for light management, and often results in out-of-equilibrium systems. Thus, in spite of the appeal of self-assembly, these features constitute a limit for the exploitation of these methods for practical applications.

Solid-state dewetting is a valid alternative to build $\mathrm{dHU}$ architectures as it provides hard materials with large dielectric constant with size tuneability spanning more than 3 orders of magnitude ${ }^{9}$. However, it is limited to few materials and requires epitaxial growth and expensive silicon on insulator wafers that for instance are not transparent at visible frequencies and are provided in a limited set of specifications (e.g. SOI, BOX and handle thickness). In addition to this, SiGe compounds feature strong absorption losses at near-UV frequencies, rendering them less adapted to applications at short wavelength. All these issues limit the range of exploitability of this self-assembly approach. 
The method proposed here enjoys all the flexibility of sol-gel chemistry, allowing to print a plethora of metal oxides ${ }^{35}$. We showed two prototypical cases of materials relevant to applications such as Silica and Titania, having a low and high refractive index respectively, and sharing reduced absorption at near-UV frequency. Nonetheless, it is possible to tune at will the refractive index (e.g., by mixing them ${ }^{56}$ or by changing their porosity ${ }^{57}$ ), and introduce nanoparticles or organic moieties ${ }^{58}$. It is possible to print on large surfaces up to 8 inches ${ }^{12,59}$, on metals, plastic, semiconductors and glasses. Thus, provided an appropriate master with ad-hoc morphologies, it is possible to reproduce it hundreds of times by framing several polymeric mastermolds from it. Note that, provided the sol-gel solutions and the mold, the overall process of softNIL can be done in less than 30 minutes in a research laboratory, independently from the sample size. All these features render our solution for dHU metasurfaces scalable to large surfaces and appealing for realistic applications.

A full assessment of the optical properties of these metal oxides-based dHU nanoarchitectures goes beyond the aim of this work. However, our results support the anti-reflective effect obtained with this kind of materials, as also recently reported by other groups ${ }^{15,22}$. Thus, the ARC effects shown here are potentially important for devices obtained via sol-NIL, especially considering the scalability of the dewetting approach to produce hard masters and the ease of implementation of the overall process. Note that there was no optimization of the ARC properties. It is also worth stressing that, differently from similar 3D ARCs based on dielectric structures ${ }^{53}$, here we avoid any top-down etching of the Si crystal as we directly print the structures in a single NIL step atop the flat Si surface. This avoids any introduction of surface defects that can be detrimental for an efficient light-to-electricity conversion in photovoltaic cells. At the same time, differently from ARCs on Si based on SiGe deposition and dewetting at high temperature ${ }^{52}$, the 
temperature budget for soft-NIL can be as low as $450^{\circ} \mathrm{C}^{12,35,60}$ rendering our process potentially back-end C-MOS compatible ${ }^{61}$. Sol-gel deposition of 2D layers of metal-oxides is a common approach to produce anti reflection coatings see for instance ${ }^{62}$ for the cases of glass and ${ }^{63}$ for the case of silicon. Here we provided a direct quantitative comparison of dHU ARC with the flat counterpart made with the same materials and nominal thickness showing an overall better performance of 3D structures with respect to the 2D counterpart.

\section{CONCLUSION}

In conclusion, we showed a simple and versatile approach based on sol-gel coatings and nano-imprint lithography of metal oxides to replicate disordered hyperuniform patterns on glass and silicon wafers. The strength of our approach is manifold: i) it is scalable as it is based on masters obtained via spontaneous dewetting and is compatible with industrial processing (such as roll-to-roll), ii) it can be transferred in a plethora of materials which density and refractive index can be tuned at will and iii) can be further functionalized adding other particles, iv) it can reproduce fine features ranging from $\sim 10 \mathrm{~nm}$ to few $\mathrm{nm}$. Thus, the importance of our results relies in the new possibility to exploit sol-gel coatings and nano-imprint lithography of metal oxides in the framework of disordered hyperuniform coatings, providing a solid ground to exploit this emerging class of materials to build functional metasurfaces.

\section{ASSOCIATED CONTENT}

Supporting Information. In the supplementary Information we provide the following data:

- Figure S.I. 1: A spectroscopic study of total diffusion by a flat 2D layer 
- Figure S.I. 2: A scheme of the method used for the fabrication of a negative replica of a SiGe master.

- Figure S.I. 3: Examples of positive and negative replicas made of $\mathrm{TiO}_{2}$ from a master bearing ordered structures.

- Figure S.I. 4: Experimentally measured bidirectional reflectance distribution function for different laser beam incidence angle.

- Figure S.I. 5: AFM investigation of titania replicas of the same master (100 nm dewetted Ge) while changing the initial thickness of the sol-gel layer from 70 to $147 \mathrm{~nm}$

- Figure S.I.6: AFM investigation of titania replicas of the same master (100 nm dewetted $\mathrm{SiGe})$ while changing the initial thickness of the sol-gel layer from 20 to $65 \mathrm{~nm}$

- Figure S.I.7: AFM images of $70 \mathrm{~nm}$ thick $\mathrm{TiO}_{2}$ replicas topped with 50, $100 \mathrm{~nm}$ and $150 \mathrm{~nm}$ of $\mathrm{SiO}_{2}$.

- Figure S.I.8: Simulation of light reflection by a flat titania layer $70 \mathrm{~nm}$ thick topped with a $\mathrm{SiO}_{2}$ layer of $150 \mathrm{~nm}$.

- Table S.I. 1: NIL of dHU with different morphology, material, size and substrate.

\section{AUTHOR INFORMATION}

\section{Corresponding Author}

*Marco Abbarci - Aix Marseille Univ, Université de Toulon, CNRS, IM2NP Marseille, France ; https://orcid.org/0000-0002-2760-4766;

Email: marco.abbarchi@im2np.fr 


\section{Authors}

Zeinab Chehadi- Aix Marseille Univ, Université de Toulon, CNRS, IM2NP Marseille, France ; https://orcid.org/0000-0002-1693-5439;

Mohammed Bouabdellaoui- Aix Marseille Univ, Université de Toulon, CNRS, IM2NP

Marseille, France ; https://orcid.org/0000-0003-0980-4104;

Mehrnaz Modaresialam- Aix Marseille Univ, Université de Toulon, CNRS, IM2NP Marseille,

France ; https://orcid.org/0000-0001-8568-8323;

Thomas Bottein- Aix Marseille Univ, Université de Toulon, CNRS, IM2NP Marseille, France ; https://orcid.org/0000-0003-0312-2057;

Marco Salvalaglio- Institute of Scientific Computing, TU Dresden, 01062 Dresden, Germany, Dresden Center for Computational Materials Science (DCMS), TU Dresden, 01062 Dresden, Germany; https://orcid.org/0000-0002-4217-0951;

Monica Bollani - Istituto di Fotonica e Nanotecnologie-Consiglio Nazionale delle Ricerche, Laboratory for Nanostructure Epitaxy and Spintronics on Silicon, Via Anzani 42, 22100 Como, Italy; https://orcid.org/0000-0002-0078-5085;

David Grosso- Aix Marseille Univ, Université de Toulon, CNRS, IM2NP Marseille, France ; https://orcid.org/0000-0002-9156-6848;

\section{Present Addresses}

$\uparrow$ Thomas Bottein's present address: Carbon Waters 14 avenue Pey Berland 33607 Pessac France Author Contributions 
The manuscript was written through contributions of all authors. All authors have given approval to the final version of the manuscript. $\ddagger$ These authors contributed equally. (match statement to author names with a symbol)

\section{Funding Sources}

We acknowledge funding provided by CNRS, UPMC and IUF (Institute University of France), the PRCI network ULYSSES (no. ANR-15-CE24-0027-01) funded by the French ANR agency, the A*MIDEX foundation (ANR-11-IDEX-0001-02), the A*MIDEX project TITANIDE (no. AM-AAP-EI-17-58-170228-16.21-ABBARCHI-SAT), and the FET-OPEN project NARCISO (no. 828890).

\section{ACKNOWLEDGMENT}

We acknowledge the facilities of the NANOTECMAT platform at the IM2NP Institute. We thank J. B. Claude and J. Wenger of Fresnel Institute for support in electronic imaging.

\section{ABBREVIATIONS}

dHU, Disordered hyperuniform.

PDMS, polydimethylsiloxane.

POTS, 1 H, 1 H, 2 H, 2 H-perfluorooctyltriethoxysilane

\section{REFERENCES}

(1) Torquato, S.; Stillinger, F. H. Local Density Fluctuations, Hyperuniformity, and Order Metrics. Phys. Rev. E 2003, 68 (4), 041113.

(2) Donev, A.; Stillinger, F. H.; Torquato, S. Unexpected Density Fluctuations in Jammed Disordered Sphere Packings. Phys. Rev. Lett. 2005, 95 (9), 090604.

(3) Kurita, R.; Weeks, E. R. Incompressibility of Polydisperse Random-Close-Packed Colloidal Particles. Phys. Rev. E 2011, 84 (3), 030401.

(4) Lesanovsky, I.; Garrahan, J. P. Out-of-Equilibrium Structures in Strongly Interacting Rydberg Gases with Dissipation. Phys. Rev. A 2014, 90 (1), 011603. 
(5) Mitchell, N. P.; Nash, L. M.; Hexner, D.; Turner, A. M.; Irvine, W. T. M. Amorphous Topological Insulators Constructed from Random Point Sets. Nature Phys 2018, 14 (4), 380-385.

(6) Gerasimenko, Y. A.; Vaskivskyi, I.; Litskevich, M.; Ravnik, J.; Vodeb, J.; Diego, M.; Kabanov, V.; Mihailovic, D. Quantum Jamming Transition to a Correlated Electron Glass in $1 \mathrm{~T}-\mathrm{TaS}_{2}$. Nat. Mater. 2019, 18 (10), 1078-1083.

(7) Hexner, D.; Levine, D. Hyperuniformity of Critical Absorbing States. Phys. Rev. Lett. 2015, 114 (11), 110602.

(8) Zhang, G.; Stillinger, F. H.; Torquato, S. Ground States of Stealthy Hyperuniform Potentials: I. Entropically Favored Configurations. Phys. Rev. E 2015, 92 (2), 022119.

(9) Salvalaglio, M.; Bouabdellaoui, M.; Bollani, M.; Benali, A.; Favre, L.; Claude, J.-B.; Wenger, J.; de Anna, P.; Intonti, F.; Voigt, A.; Abbarchi, M. Hyperuniform Monocrystalline Structures by Spinodal Solid-State Dewetting. Phys. Rev. Lett. 2020, 125 (12), 126101.

(10) Jiao, Y.; Lau, T.; Hatzikirou, H.; Meyer-Hermann, M.; Joseph C. Corbo; Torquato, S. Avian Photoreceptor Patterns Represent a Disordered Hyperuniform Solution to a Multiscale Packing Problem. Phys. Rev. E 2014, 89 (2), 022721.

(11) Caselli, N.; Riboli, F.; Intonti, F.; La China, F.; Biccari, F.; Gerardino, A.; Gurioli, M. Spatial Steadiness of Individual Disorder Modes upon Controlled Spectral Tuning. APL Photonics 2016, 1 (4), 041301.

(12) Bochet-Modaresialam, M.; Claude, J.-B.; Grosso, D.; Abbarchi, M. Methylated Silica Surfaces Having Tapered Nipple-Dimple Nanopillar Morphologies as Robust Broad-Angle and Broadband Antireflection Coatings. ACS Appl. Nano Mater. 2020, 3 (6), 5231-5239.

(13) Zhou, W.; Cheng, Z.; Zhu, B.; Sun, X.; Tsang, H. K. Hyperuniform Disordered Network Polarizers. IEEE J. Select. Topics Quantum Electron. 2016, 22 (6), 288-294.

(14) Degl'Innocenti, R.; Shah, Y. D.; Masini, L.; Ronzani, A.; Pitanti, A.; Ren, Y.; Jessop, D. S.; Tredicucci, A.; Beere, H. E.; Ritchie, D. A. Hyperuniform Disordered Terahertz Quantum Cascade Laser. Sci Rep 2016, 6 (1), 19325.

(15) Zhang, H.; Chu, H.; Giddens, H.; Wu, W.; Hao, Y. Experimental Demonstration of Luneburg Lens Based on Hyperuniform Disordered Media. Appl. Phys. Lett. 2019, 114 (5), 053507.

(16) Florescu, M.; Torquato, S.; Steinhardt, P. J. Designer Disordered Materials with Large, Complete Photonic Band Gaps. Proceedings of the National Academy of Sciences 2009, 106 (49), 20658-20663.

(17) Man, W.; Florescu, M.; Williamson, E. P.; He, Y.; Hashemizad, S. R.; Leung, B. Y. C.; Liner, D. R.; Torquato, S.; Chaikin, P. M.; Steinhardt, P. J. Isotropic Band Gaps and Freeform Waveguides Observed in Hyperuniform Disordered Photonic Solids. Proceedings of the National Academy of Sciences 2013, 110 (40), 15886-15891.

(18) Muller, N.; Haberko, J.; Marichy, C.; Scheffold, F. Silicon Hyperuniform Disordered Photonic Materials with a Pronounced Gap in the Shortwave Infrared. Advanced Optical Materials 2014, 2 (2), 115-119.

(19) Froufe-Pérez, L. S.; Engel, M.; Damasceno, P. F.; Muller, N.; Haberko, J.; Glotzer, S. C.; Scheffold, F. Role of Short-Range Order and Hyperuniformity in the Formation of Band Gaps in Disordered Photonic Materials. Phys. Rev. Lett. 2016, 117 (5), 053902.

(20) Castro-Lopez, M.; Gaio, M.; Sellers, S.; Gkantzounis, G.; Florescu, M.; Sapienza, R. Reciprocal Space Engineering with Hyperuniform Gold Disordered Surfaces. APL Photonics 2017, 2 (6), 061302. 
(21) Piechulla, P. M.; Fuhrmann, B.; Slivina, E.; Rockstuhl, C.; Wehrspohn, R. B.; Sprafke, A. N. Tailored Light Scattering through Hyperuniform Disorder in Self-Organized Arrays of High-Index Nanodisks. Adv. Optical Mater. 2021, 2100186.

(22) Tavakoli, N.; Spalding, R.; Koppejan, P.; Gkantzounis, G.; Wang, C.; Röhrich, R.; Kontoleta, E.; Koenderink, A. F.; Sapienza, R.; Florescu, M.; Alarcon-Llado, E. Over 65\% Sunlight Absorption in a $1 \mathrm{Mm}$ Si Slab with Hyperuniform Texture; preprint; In Review, 2020.

(23) Weijs, J. H.; Jeanneret, R.; Dreyfus, R.; Bartolo, D. Emergent Hyperuniformity in Periodically Driven Emulsions. Phys. Rev. Lett. 2015, 115 (10), 108301.

(24) Zito, G.; Rusciano, G.; Pesce, G.; Malafronte, A.; Di Girolamo, R.; Ausanio, G.; Vecchione, A.; Sasso, A. Nanoscale Engineering of Two-Dimensional Disordered Hyperuniform Block-Copolymer Assemblies. Phys. Rev. E 2015, 92 (5), 050601.

(25) Goldfriend, T.; Diamant, H.; Witten, T. A. Screening, Hyperuniformity, and Instability in the Sedimentation of Irregular Objects. Phys. Rev. Lett. 2017, 118 (15), 158005.

(26) Wang, J.; Schwarz, J. M.; Paulsen, J. D. Hyperuniformity with No Fine Tuning in Sheared Sedimenting Suspensions. Nat Commun 2018, 9 (1), 2836.

(27) Becker, J.; Grün, G.; Seemann, R.; Mantz, H.; Jacobs, K.; Mecke, K. R.; Blossey, R. Complex Dewetting Scenarios Captured by Thin-Film Models. Nature Mater 2003, 2 (1), 59-63.

(28) Stoop, N.; Lagrange, R.; Terwagne, D.; Reis, P. M.; Dunkel, J. Curvature-Induced Symmetry Breaking Determines Elastic Surface Patterns. Nature Mater 2015, 14 (3), 337342.

(29) Cahn, J. W. On Spinodal Decomposition. Acta Metallurgica 1961, 9 (9), 795-801.

(30) Ma, Z.; Torquato, S. Random Scalar Fields and Hyperuniformity. Journal of Applied Physics 2017, 121 (24), 244904.

(31) Ma, Z.; Lomba, E.; Torquato, S. Optimized Large Hyperuniform Binary Colloidal Suspensions in Two Dimensions. Phys. Rev. Lett. 2020, 125 (6), 068002.

(32) Benali, A.; Claude, J.-B.; Granchi, N.; Checcucci, S.; Bouabdellaoui, M.; Zazoui, M.; Bollani, M.; Salvalaglio, M.; Wenger, J.; Favre, L.; Grosso, D.; Ronda, A.; Berbezier, I.; Gurioli, M.; Abbarchi, M. Flexible Photonic Devices Based on Dielectric Antennas. J. Phys. Photonics 2020, 2 (1), 015002.

(33) Naffouti, M.; Backofen, R.; Salvalaglio, M.; Bottein, T.; Lodari, M.; Voigt, A.; David, T.; Benkouider, A.; Fraj, I.; Favre, L.; Ronda, A.; Berbezier, I.; Grosso, D.; Abbarchi, M.; Bollani, M. Complex Dewetting Scenarios of Ultrathin Silicon Films for Large-Scale Nanoarchitectures. Sci. Adv. 2017, 3 (11), eaao1472.

(34) Abbarchi, M.; Naffouti, M.; Lodari, M.; Salvalaglio, M.; Backofen, R.; Bottein, T.; Voigt, A.; David, T.; Claude, J.-B.; Bouabdellaoui, M.; Benkouider, A.; Fraj, I.; Favre, L.; Ronda, A.; Berbezier, I.; Grosso, D.; Bollani, M. Solid-State Dewetting of Single-Crystal Silicon on Insulator: Effect of Annealing Temperature and Patch Size. Microelectronic Engineering 2018, 190, 1-6.

(35) Bottein, T.; Dalstein, O.; Putero, M.; Cattoni, A.; Faustini, M.; Abbarchi, M.; Grosso, D. Environment-Controlled Sol-Gel Soft-NIL Processing for Optimized Titania, Alumina, Silica and Yttria-Zirconia Imprinting at Sub-Micron Dimensions. Nanoscale 2018, 10 (3), 1420-1431.

(36) Loscutoff, P. W.; Bent, S. F. REACTIVITY OF THE GERMANIUM SURFACE: Chemical Passivation and Functionalization. Annu. Rev. Phys. Chem. 2006, 57 (1), 467-495. 
(37) Mui, C.; Han, J. H.; Wang, G. T.; Musgrave, C. B.; Bent, S. F. Proton Transfer Reactions on Semiconductor Surfaces. J. Am. Chem. Soc. 2002, 124 (15), 4027-4038.

(38) Grosso, D. How to Exploit the Full Potential of the Dip-Coating Process to Better Control Film Formation. J. Mater. Chem. 2011, 21 (43), 17033.

(39) Germer, T. A.; Zwinkels, J. C.; Tsai, B. K. Spectrophotometry: Accurate Measurement of Optical Properties of Materials; Elsevier Science: Amsterdam, 2014.

(40) Loizillon, J.; Baumgartner, B.; Sinturel, C.; Abbarchi, M.; Lendl, B.; Grosso, D. In-Depth Study of Coating Multimodal Porosity Using Ellipsometry Porosimetry in Desorption Scanning Mode. J. Phys. Chem. C 2019, 123 (38), 23464-23479.

(41) Boissiere, C.; Grosso, D.; Lepoutre, S.; Nicole, L.; Bruneau, A. B.; Sanchez, C. Porosity and Mechanical Properties of Mesoporous Thin Films Assessed by Environmental Ellipsometric Porosimetry. Langmuir 2005, 21 (26), 12362-12371.

(42) Bottein, T.; Wood, T.; David, T.; Claude, J. B.; Favre, L.; Berbézier, I.; Ronda, A.; Abbarchi, M.; Grosso, D. "Black" Titania Coatings Composed of Sol-Gel Imprinted Mie Resonators Arrays. Adv. Funct. Mater. 2017, 27 (2), 1604924.

(43) Checcucci, S.; Bottein, T.; Gurioli, M.; Favre, L.; Grosso, D.; Abbarchi, M. Multifunctional Metasurfaces Based on Direct Nanoimprint of Titania Sol-Gel Coatings. Advanced Optical Materials 2019, 7 (10), 1801406.

(44) Grosso, D.; Boissière, C.; Sanchez, C. Ultralow-Dielectric-Constant Optical Thin Films Built from Magnesium Oxyfluoride Vesicle-like Hollow Nanoparticles. Nature Mater 2007, $6(8), 572-575$.

(45) Gitlin, L.; Schulze, P.; Belder, D. Rapid Replication of Master Structures by Double Casting with PDMS. Lab Chip 2009, 9 (20), 3000.

(46) Mutreja, I.; Woodfield, T. B. F.; Sperling, S.; Nock, V.; Evans, J. J.; Alkaisi, M. M. Positive and Negative Bioimprinted Polymeric Substrates: New Platforms for Cell Culture. Biofabrication 2015, 7 (2), 025002.

(47) He, R.; Xiao, J.; Zhang, M.; Zhang, Z.; Zhang, W.; Cao, Y.; Liu, Y.; Chen, Y. Artificial Honeycomb-Inspired TiO2 Nanorod Arrays with Tunable Nano/Micro Interfaces for Improving Poly(Dimethylsiloxane) Surface Hydrophobicity. J Mater Sci 2016, 51 (6), 2935-2941.

(48) Xie, R.; Long, G. G.; Weigand, S. J.; Moss, S. C.; Carvalho, T.; Roorda, S.; Hejna, M.; Torquato, S.; Steinhardt, P. J. Hyperuniformity in Amorphous Silicon Based on the Measurement of the Infinite-Wavelength Limit of the Structure Factor. Proceedings of the National Academy of Sciences 2013, 110 (33), 13250-13254.

(49) Wilken, S.; Guerra, R. E.; Pine, D. J.; Chaikin, P. M. Hyperuniform Structures Formed by Shearing Colloidal Suspensions. Phys. Rev. Lett. 2020, 125 (14), 148001.

(50) de Graff, A. M. R.; Thorpe, M. F. The Long-Wavelength Limit of the Structure Factor of Amorphous Silicon and Vitreous Silica. Acta Crystallogr A Found Crystallogr 2010, 66 (1), 22-31.

(51) Castro-Lopez, M.; Gaio, M.; Sellers, S.; Gkantzounis, G.; Florescu, M.; Sapienza, R. Reciprocal Space Engineering with Hyperuniform Gold Disordered Surfaces. APL Photonics 2017, 2 (6), 061302.

(52) Bouabdellaoui, M.; Checcucci, S.; Wood, T.; Naffouti, M.; Sena, R. P.; Liu, K.; Ruiz, C. M.; Duche, D.; le Rouzo, J.; Escoubas, L.; Berginc, G.; Bonod, N.; Zazoui, M.; Favre, L.; Metayer, L.; Ronda, A.; Berbezier, I.; Grosso, D.; Gurioli, M.; Abbarchi, M. Self- 
Assembled Antireflection Coatings for Light Trapping Based on SiGe Random Metasurfaces. Phys. Rev. Materials 2018, 2 (3), 035203.

(53) Spinelli, P.; Verschuuren, M. A.; Polman, A. Broadband Omnidirectional Antireflection Coating Based on Subwavelength Surface Mie Resonators. Nat Commun 2012, 3 (1), 692.

(54) Checcucci, S.; Bottein, T.; Claude, J.-B.; Wood, T.; Putero, M.; Favre, L.; Gurioli, M.; Abbarchi, M.; Grosso, D. Titania-Based Spherical Mie Resonators Elaborated by HighThroughput Aerosol Spray: Single Object Investigation. Adv. Funct. Mater. 2018, 28 (31), 1801958.

(55) Weijs, J. H.; Bartolo, D. Mixing by Unstirring: Hyperuniform Dispersion of Interacting Particles upon Chaotic Advection. Phys. Rev. Lett. 2017, 119 (4), 048002.

(56) Louis, B.; Krins, N.; Faustini, M.; Grosso, D. Understanding Crystallization of Anatase into Binary $\mathrm{SiO}_{2} / \mathrm{TiO}_{2}$ Sol-Gel Optical Thin Films: An in Situ Thermal Ellipsometry Analysis. J. Phys. Chem. C 2011, 115 (7), 3115-3122.

(57) Li, R.; Boudot, M.; Boissière, C.; Grosso, D.; Faustini, M. Suppressing Structural Colors of Photocatalytic Optical Coatings on Glass: The Critical Role of $\mathrm{SiO}_{2}$. ACS Appl. Mater. Interfaces 2017, 9 (16), 14093-14102.

(58) Sanchez, C.; Boissière, C.; Grosso, D.; Laberty, C.; Nicole, L. Design, Synthesis, and Properties of Inorganic and Hybrid Thin Films Having Periodically Organized Nanoporosity. Chem. Mater. 2008, 20 (3), 682-737.

(59) Zhang, W.; Tu, J.; Long, W.; Lai, W.; Sheng, Y.; Guo, T. Preparation of SiO 2 AntiReflection Coatings by Sol-Gel Method. Energy Procedia 2017, 130, 72-76.

(60) Dalstein, O.; Ceratti, D. R.; Boissière, C.; Grosso, D.; Cattoni, A.; Faustini, M. Nanoimprinted, Submicrometric, MOF-Based 2D Photonic Structures: Toward Easy Selective Vapors Sensing by a Smartphone Camera. Adv. Funct. Mater. 2016, 26 (1), 8190.

(61) Lee, Y. H. D.; Lipson, M. Back-End Deposited Silicon Photonics for Monolithic Integration on CMOS. IEEE J. Select. Topics Quantum Electron. 2013, 19 (2), 8200207-8200207.

(62) Prado, R.; Beobide, G.; Marcaide, A.; Goikoetxea, J.; Aranzabe, A. Development of Multifunctional Sol-Gel Coatings: Anti-Reflection Coatings with Enhanced Self-Cleaning Capacity. Solar Energy Materials and Solar Cells 2010, 94 (6), 1081-1088.

(63) San Vicente, G.; Morales, A.; Gutierrez, M. T. Preparation and Characterization of Sol-Gel $\mathrm{TiO}_{2}$ Antireflective Coatings for Silicon. Thin Solid Films 2001, 391 (1), 133-137. 


\section{Scalable Disordered Hyperuniform Architectures}

\section{Via Nano-Imprint Lithography Of Metal Oxides}

Zeinab Chehadi ${ }^{1}$, Mohammed Bouabdellaoui ${ }^{1}$, Mehrnaz Modaresialam ${ }^{1}$, Thomas Bottein ${ }^{1, \dagger}$,

Marco Salvalaglio ${ }^{2,3}$, Monica Bollani ${ }^{4}$, David Grosso $^{1}$, and Marco Abbarchi, ${ }^{1, *}$

${ }^{1}$ Aix Marseille Univ, Université de Toulon, CNRS, IM2NP Marseille, France

${ }^{2}$ Institute of Scientific Computing, TU Dresden, 01062 Dresden, Germany

${ }^{3}$ Dresden Center for Computational Materials Science (DCMS), TU Dresden, 01062 Dresden,

Germany

${ }^{4}$ Istituto di Fotonica e Nanotecnologie-Consiglio Nazionale delle Ricerche, Laboratory for

Nanostructure Epitaxy and Spintronics on Silicon, Via Anzani 42, 22100 Como, Italy

*Corresponding author: marco.abbarchi@im2np.fr 


\section{Optical quality thin layers deposited via sol-gel dip coating}

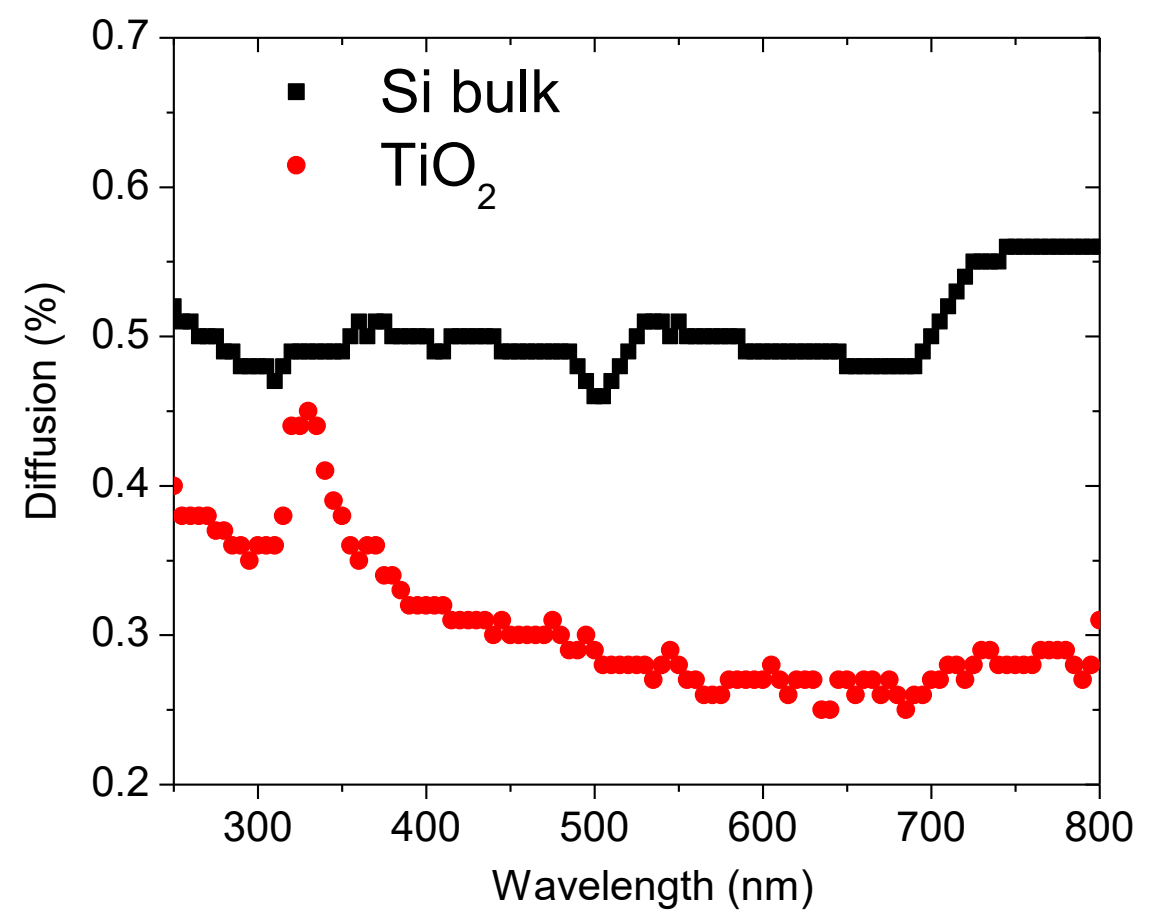

Figure S.I. 1. Light scattering at near UV and visible frequency for a $\mathrm{Si}$ wafer and for a $\mathrm{TiO}_{2}$ layer deposited via sol gel.

\section{Inverted structures, negative replicas}

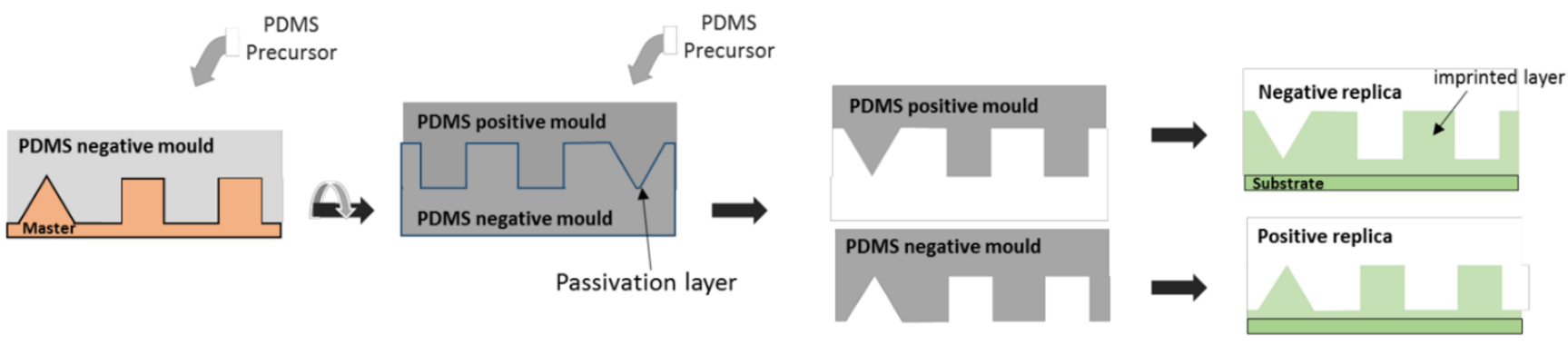

Figure S.I. 2. Scheme representing the principle of inverted PDMS molds. First, a mould is made from an initial master by pouring the PDMS reactants and curing it at $70^{\circ} \mathrm{C}$ for $1 \mathrm{~h}$ before releasing from the master. This negative mould displays the inverse of the master features. Secondly, after passivation of its surface, the negative mould is used as a master, yielding a positive mould with the same features than the initial master. 

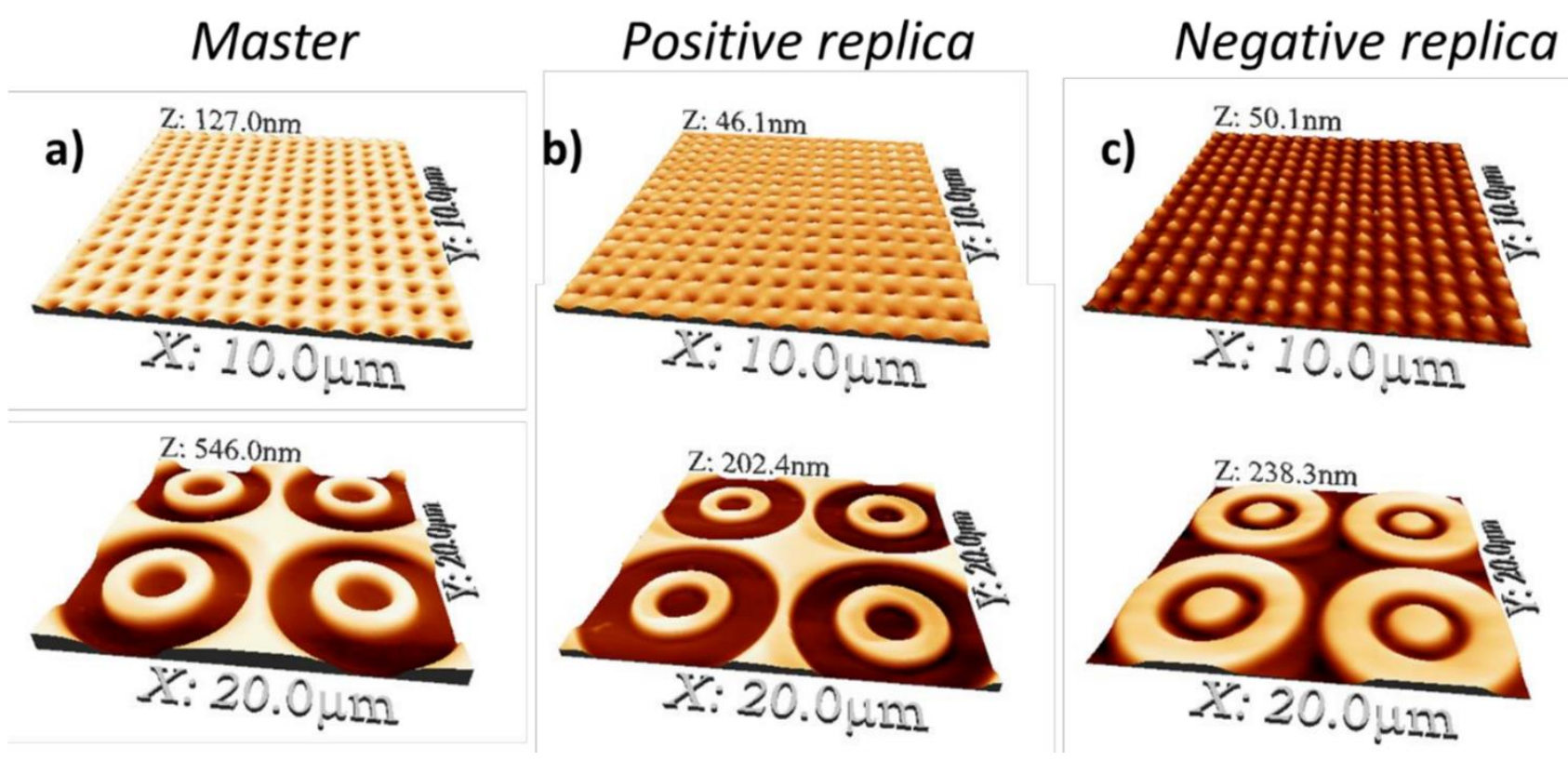

Figure S.I. 3. (a) Starting hard masters obtained via focused on beam etching of a bulk Si wafer. Top panel, holes. Bottom panels: rings. (b) Positive $\mathrm{TiO}_{2}$ replicas. (c) Negative $\mathrm{TiO}_{2}$ replicas.

\begin{tabular}{|c|c|c|c|c|}
\hline \multicolumn{5}{|l|}{ HOLES } \\
\hline UNITS: nm & \multicolumn{2}{|c|}{ Depth $( \pm 5 \mathrm{~nm})$} & $\begin{array}{l}\text { Diamter }( \pm 5 \\
\mathrm{nm})\end{array}$ & Pitch $( \pm 5 \mathrm{~nm})$ \\
\hline master & \multicolumn{2}{|l|}{100} & 270 & 700 \\
\hline Positive replica & \multicolumn{2}{|l|}{28} & 285 & 700 \\
\hline Negative replica & \multicolumn{2}{|l|}{30} & 285 & 700 \\
\hline \multicolumn{5}{|l|}{ RINGS } \\
\hline UNITS: $\mu \mathrm{m}$ & $\begin{array}{l}\text { Height }( \pm 10 \\
\mu \mathrm{m})\end{array}$ & $\begin{array}{l}\operatorname{Dext}( \pm 0.2 \\
\mu \mathrm{m})\end{array}$ & Dint $( \pm 0.2 \mu \mathrm{m})$ & Pitch $( \pm 0.1 \mu \mathrm{m})$ \\
\hline master & 400 & 5.5 & 3 & 10 \\
\hline Positive replica & 150 & 5.1 & 2.8 & 10 \\
\hline Negative replica & 160 & 5.6 & 2.7 & 10 \\
\hline
\end{tabular}

Table S.I. 2: NIL of dHU with different morphology, material, size and substrate. 


\section{Disordered hyperuniformity of soft-NIL structures}

The supplementary Figure S.I.4 shows the bidirectional reflectance distribution of the master measured by changing the angle of incidence of the excitation from $\theta \mathrm{exc}=0$ to -40 degrees in steps of 10 degrees.

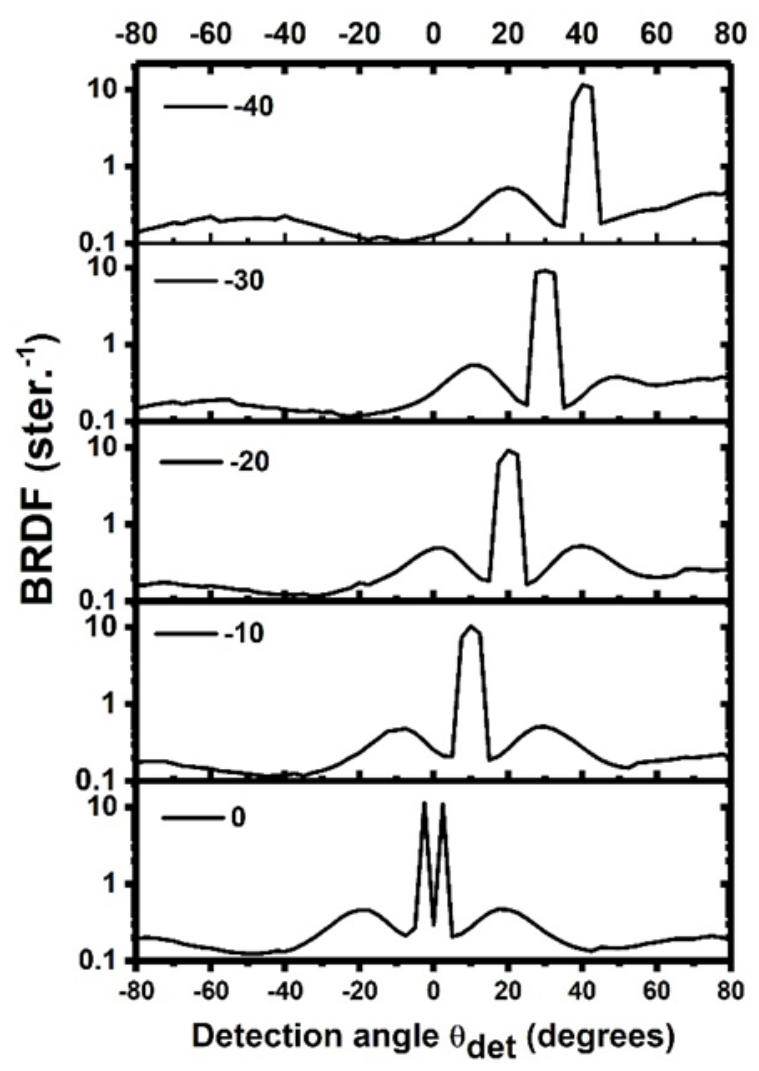

Figure SI. 4. Bidirectional reflectance distribution function (BRDF) of the master measured by changing the angle of incidence of the excitation from $\theta \mathrm{exc}=0$ to -40 degrees. 


\section{Sol-gel thickness tuning}
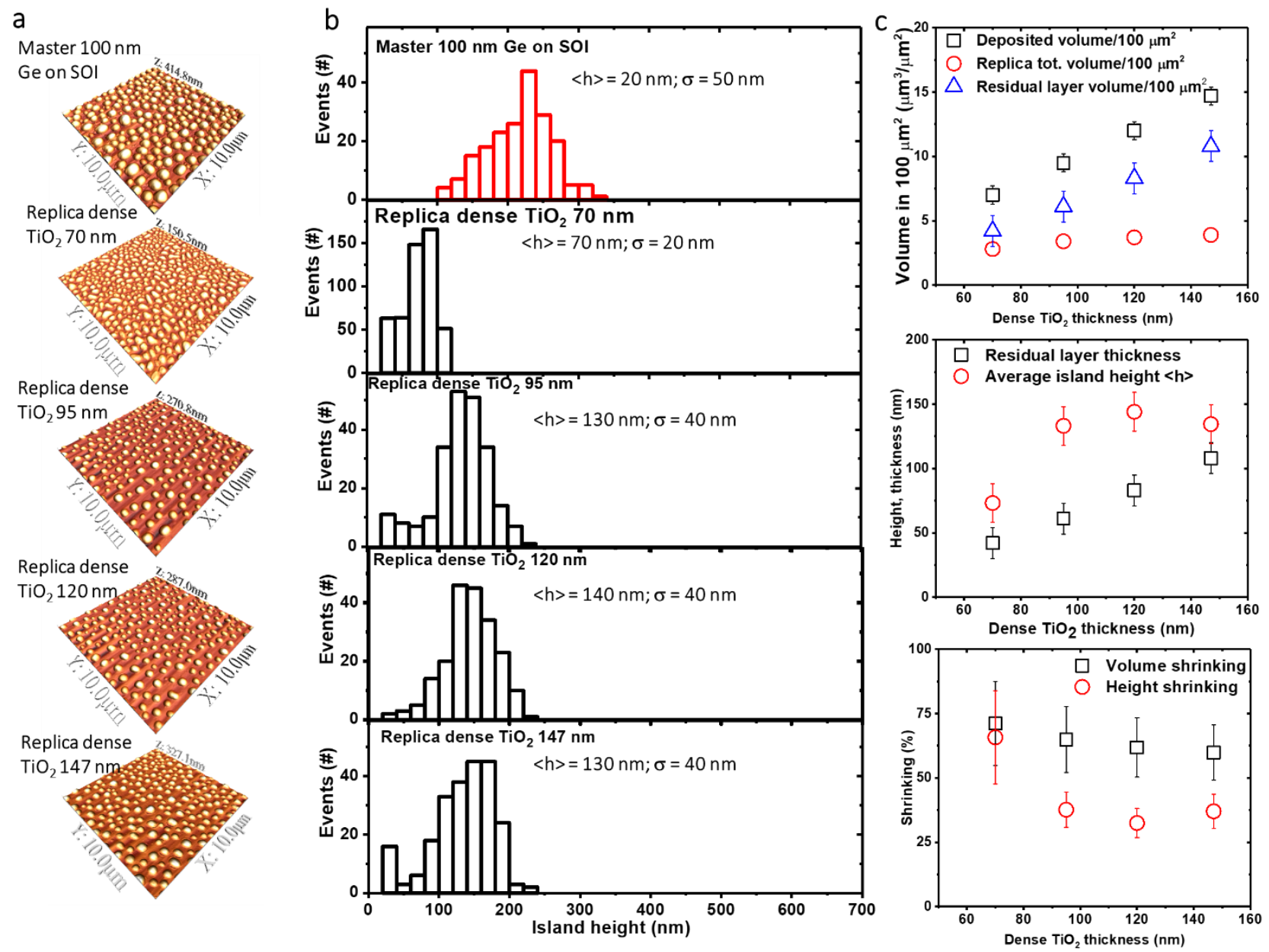

Figure SI. 5. (a) From the top to the bottom panel: AFM images of a Ge master (dewetted from a $100 \mathrm{~nm}$ thick layer) bearing isolated islands, dense $\mathrm{TiO}_{2}$ replica printed on a $70 \mathrm{~nm}, 95,120$ and $147 \mathrm{~nm}$ thick layers. (b) Statistical distribution of islands height as deduced from the atomic force microscope images in a. On each panel the average height $<\mathrm{h}>$ and corresponding standard deviation $\sigma$ are reported. (c) Top panel: deposited volume (squares), total volume of the printed structures (circles) and residual layer volume (triangles) as a function of the deposited $\mathrm{TiO}_{2}$ thickness. Central panel: average islands height $<\mathrm{h}>$ (circles) and residual layer thickness (squares). Bottom panel: height (circles) and volume shrinking of the replicas with respect to the master as a function of the deposited layer thickness. 


$$
\text { a }
$$$$
\text { Master } 100 \mathrm{~nm}
$$$$
\text { SiGe (30\%) on }{ }^{2} \text {. } 200 .
$$$$
\text { SOI }
$$

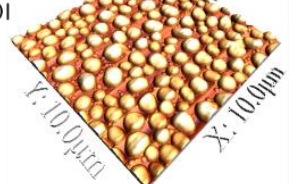

Replica dense

$\mathrm{TiO}_{2} 20 \mathrm{~nm}$

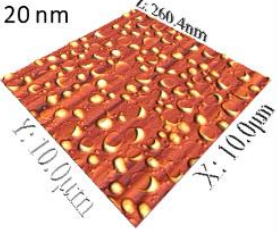

Replica dense

$\mathrm{TiO}_{2} 38 \mathrm{~nm}$

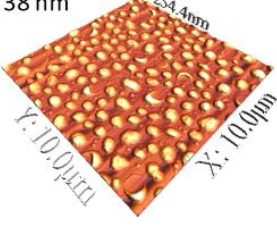

Replica dense

$\mathrm{TiO}_{2} 60 \mathrm{~nm}$

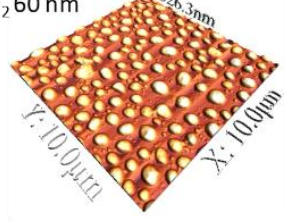

b
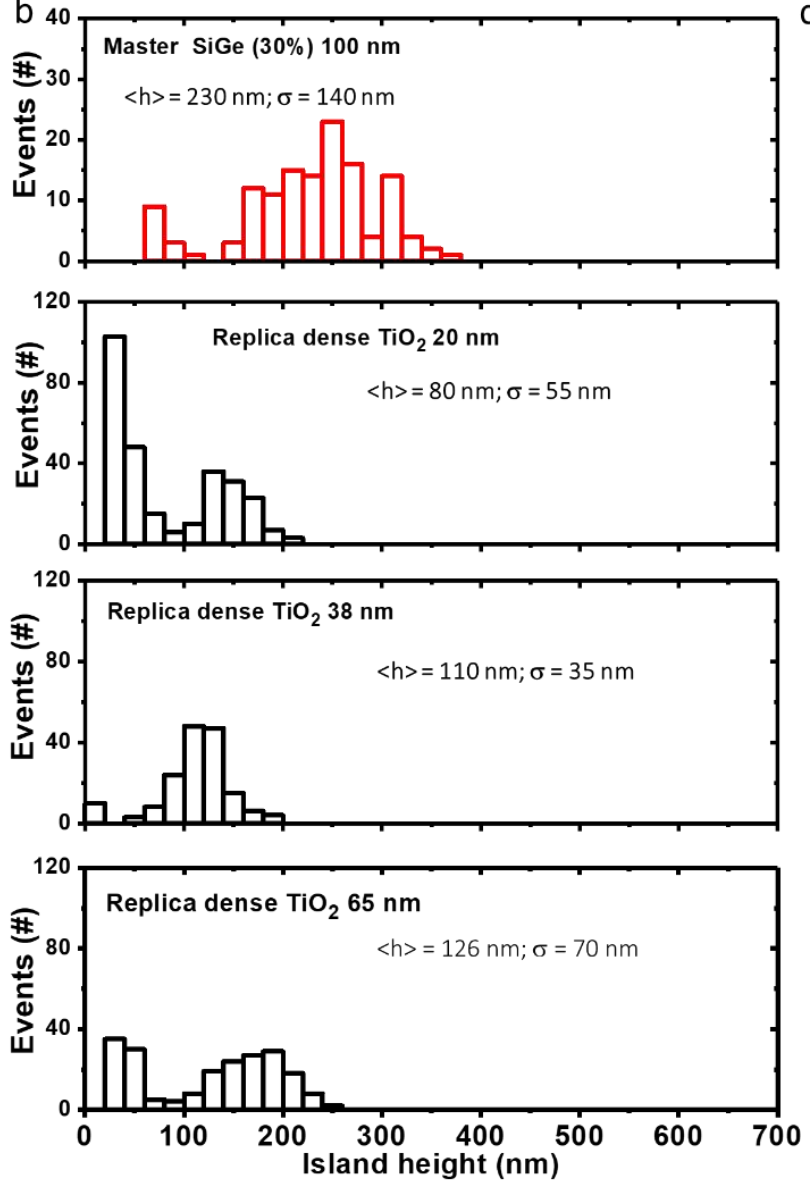
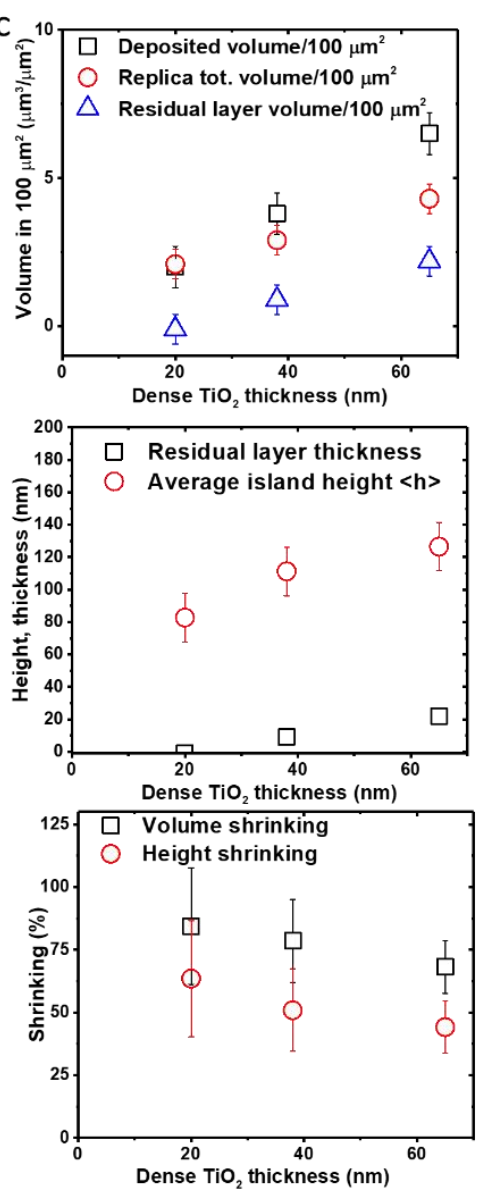

Figure SI. 6. (a) From the top to the bottom panel: AFM images of a SiGe master (dewetted from a $100 \mathrm{~nm}$ thick layer) bearing isolated islands, dense $\mathrm{TiO}_{2}$ replica printed on a 20,38, and $60 \mathrm{~nm}$ thick layers. (b) Statistical distribution of islands height as deduced from the atomic force microscope images in a. On each panel the average height $<\mathrm{h}>$ and corresponding standard deviation $\sigma$ are reported. (c) Top panel: deposited volume (squares), total volume of the printed structures (circles) and residual layer volume (triangles) as a function of the deposited $\mathrm{TiO}_{2}$ thickness. Central panel: average islands height $<\mathrm{h}>$ (circles) and residual layer thickness (squares). Bottom panel: height (circles) and volume shrinking of the replicas with respect to the master as a function of the deposited layer thickness. 


\section{Optical properties}

a
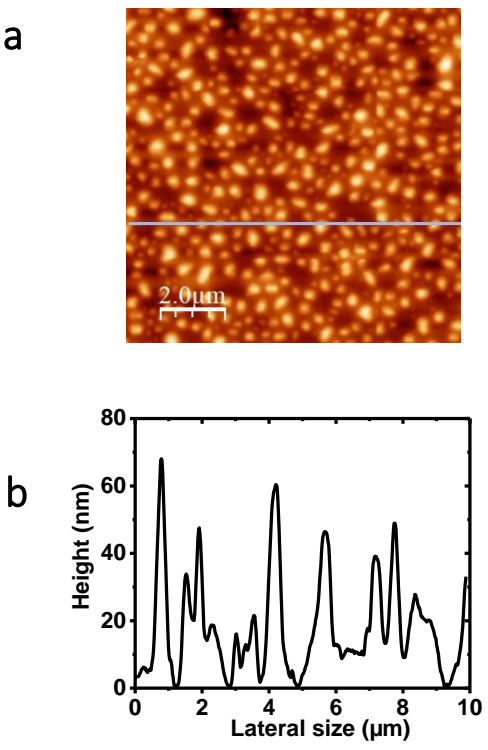

C

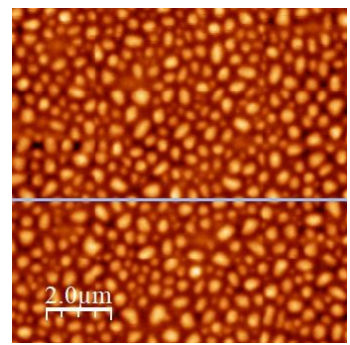

$\mathrm{e}$
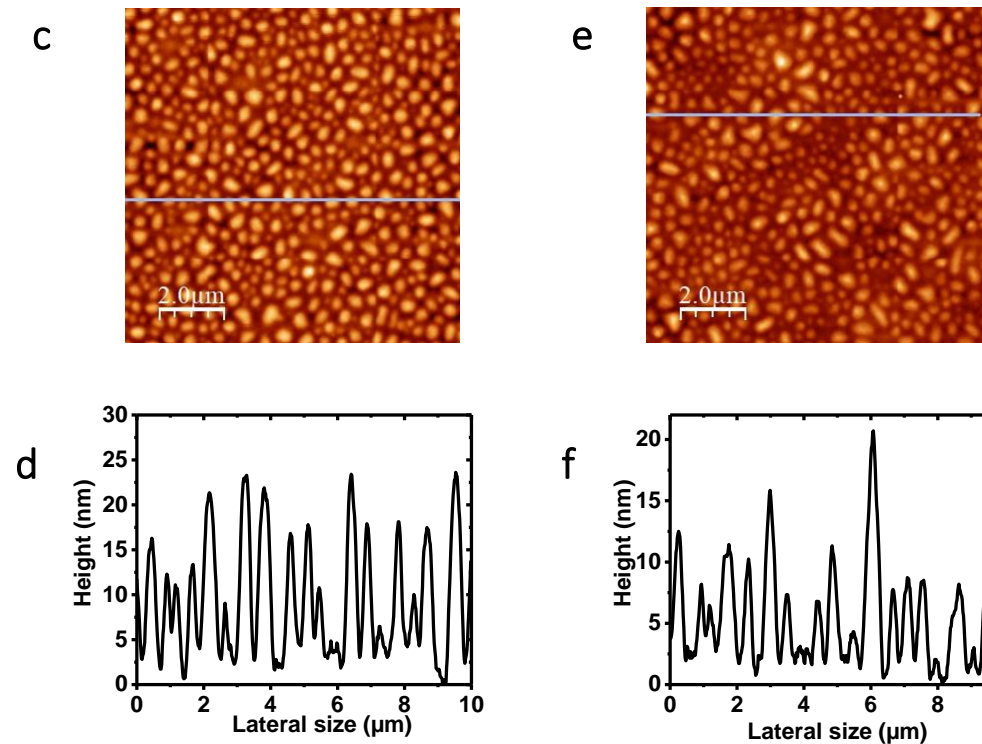

$\mathrm{f}$

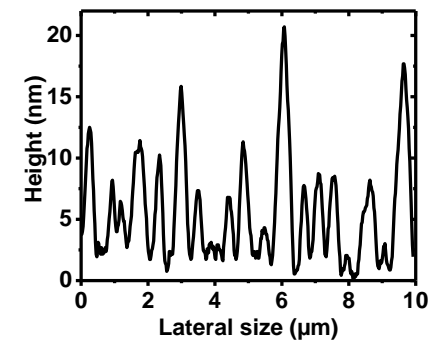

Figure SI. 7. Atomic force microscope (AFM) image for a $70 \mathrm{~nm}$ thick $\mathrm{TiO}_{2}$ replica with a top a) $50 \mathrm{~nm} \mathrm{SiO}_{2}$ c) 100 $\mathrm{nm} \mathrm{SiO}_{2}$ and e) $150 \mathrm{~nm} \mathrm{SiO}$. Height profile obtained b) from a), d) from c) and f) from e). The surface roughness average is $14 \mathrm{~nm}$ with soft-NIL of $70 \mathrm{~nm}$ of $\mathrm{TiO}_{2}$ with a top $50 \mathrm{~nm} \mathrm{SiO}{ }_{2}$ and $5 \mathrm{~nm}$ with soft-NIL of $70 \mathrm{~nm}$ of $\mathrm{TiO}_{2}$ with a top $100 \mathrm{~nm} \mathrm{SiO} 2$ and $3 \mathrm{~nm}$ with soft $-\mathrm{NIL}$ of $70 \mathrm{~nm}$ of $\mathrm{TiO}_{2}$ with a top $150 \mathrm{~nm} \mathrm{SiO}$. 


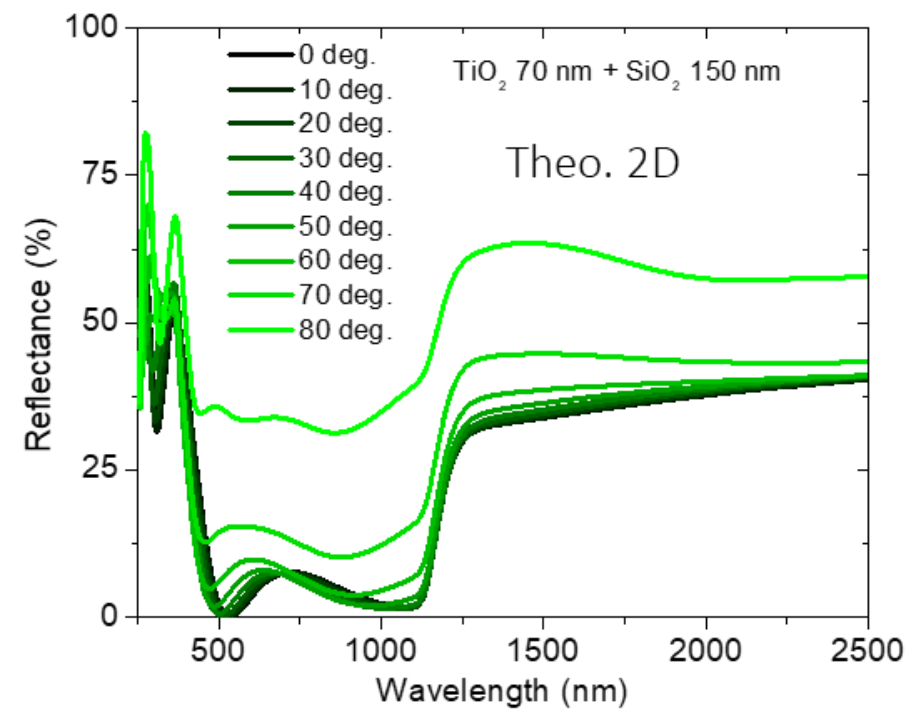

Figure SI. 8. Simulations of light reflection from a bilayer on $\mathrm{Si}$ composed of $70 \mathrm{~nm}$ of $\mathrm{TiO}_{2}$ and $150 \mathrm{~nm} \mathrm{SiO}_{2}$. The simulations show different cases relative to different incidence angles with respect to the sample normal

TOC:

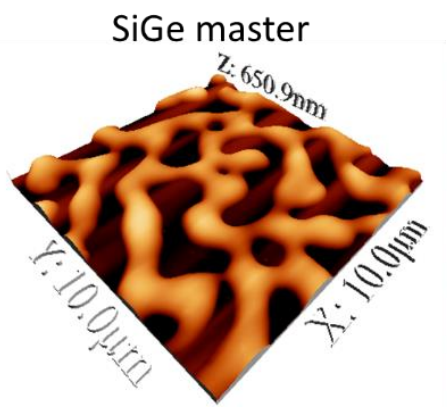

$\mathrm{TiO}_{2}$ replica

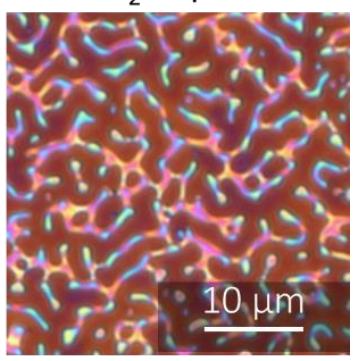

